Zhi Chen, Xianhua Tang, and Jian Zhang*

\title{
Sign-changing multi-bump solutions for the Chern-Simons-Schrödinger equations in $\mathbb{R}^{2}$
}

https://doi.org/10.1515/anona-2020-0041

Received Marh 20, 2019; accepted July 11, 2019.

Abstract: In this paper we consider the nonlinear Chern-Simons-Schrödinger equations with general nonlinearity

$$
-\Delta u+\lambda V(|x|) u+\left(\frac{h^{2}(|x|)}{|x|^{2}}+\int_{|x|}^{\infty} \frac{h(s)}{s} u^{2}(s) d s\right) u=f(u), \quad x \in \mathbb{R}^{2},
$$

where $\lambda>0, V$ is an external potential and

$$
h(s)=\frac{1}{2} \int_{0}^{s} r u^{2}(r) d r=\frac{1}{4 \pi} \int_{B_{s}} u^{2}(x) d x
$$

is the so-called Chern-Simons term. Assuming that the external potential $V$ is nonnegative continuous function with a potential well $\Omega:=\operatorname{int} V^{-1}(0)$ consisting of $k+1$ disjoint components $\Omega_{0}, \Omega_{1}, \Omega_{2} \cdots, \Omega_{k}$, and the nonlinearity $f$ has a general subcritical growth condition, we are able to establish the existence of signchanging multi-bump solutions by using variational methods. Moreover, the concentration behavior of solutions as $\lambda \rightarrow+\infty$ are also considered.

Keywords: Chern-Simons-Schrödinger equations; sign-changing solution; potential well; concentration behavior

MSC: 35J20; 58E50

\section{Introduction and main results}

In this paper we are interested in the following nonlinear Schrödinger system with the gauge field

$$
\left\{\begin{array}{l}
i D_{0} \phi+\left(D_{1} D_{1}+D_{2} D_{2}\right) \phi+g(\phi)=0, \\
\partial_{0} A_{1}-\partial_{1} A_{0}=-\operatorname{Im}\left(\bar{\phi} D_{2} \phi\right), \\
\partial_{0} A_{2}-\partial_{2} A_{0}=\operatorname{Im}\left(\bar{\phi} D_{1} \phi\right), \\
\partial_{1} A_{2}-\partial_{2} A_{1}=-\frac{1}{2}|\phi|^{2},
\end{array}\right.
$$

where $i$ denotes the imaginary unit, $\partial_{0}=\frac{\partial}{\partial t}$, $\partial_{1}=\frac{\partial}{\partial x_{1}}, \partial_{2}=\frac{\partial}{\partial_{x_{2}}}$ for $\left(t, x_{1}, x_{2}\right) \in \mathbb{R}^{1+2}, \phi: \mathbb{R}^{1+2} \rightarrow \mathbb{C}$ is the complex scalar field, $A_{\kappa}: \mathbb{R}^{1+2} \rightarrow \mathbb{R}$ is the gauge field and $D_{\kappa}=\partial_{\kappa}+i A_{\kappa}$ is the covariant derivative for $\kappa=0,1,2$. This model (1.1) was first proposed and studied in [22-24], and is sometimes called the ChernSimons-Schrödinger equations. The two-dimensional Chern-Simons-Schrödinger equations is a nonrelativistic quantum model describing the dynamics of a large number of particles in the plane, which interact both

Zhi Chen, School of Mathematics and Statistics, Central South University, Changsha, 410083 Hunan, P. R. China, E-mail: math120701012cz@163.com

Xianhua Tang, School of Mathematics and Statistics, Central South University, Changsha, 410083 Hunan, P. R. China, E-mail: tangxh@mail.csu.edu.cn

*Corresponding Author: Jian Zhang, School of Mathematics and Statistics, Hunan University of Technology and Business, Changsha, 410205 Hunan, P. R. China, E-mail: zhangjian433130@163.com 
directly and via a self-generated electromagnetic field. Moreover, it describes an external uniform magnetic field which is of great phenomenological interest for applications of Chern-Simons theory to the quantum Hall effect.

As usual in Chern-Simons theory, system (1.1) is invariant under gauge transformation

$$
\phi \rightarrow \phi e^{i \chi}, A_{\kappa} \rightarrow A_{\kappa}-\partial_{\kappa} \chi
$$

for any arbitrary $C^{\infty}$ function $\chi$. The existence of standing wave solutions for system (1.1) with power type nonlinearity, that is, $g(u)=\lambda|u|^{p-1} u(p>1$ and $\lambda>0)$, has been investigated recently by a number of authors. For example, see $[6,7,20,25,32]$ and the references therein. The standing wave solutions of system (1.1) have the following form

$$
\begin{aligned}
& \phi(t, x)=u(|x|) e^{i \omega t}, \quad A_{0}(t, x)=k(|x|), \\
& A_{1}(t, x)=\frac{x_{2}}{|x|^{2}} h(|x|), \quad A_{2}(t, x)=-\frac{x_{1}}{|x|^{2}} h(|x|),
\end{aligned}
$$

where $\omega>0$ is a given frequency, $u, k, h$ are real valued functions depending only on $|x|$. Note that the ansatz (1.2) satisfies the Coulomb gauge condition $\partial_{1} A_{1}+\partial_{2} A_{2}=0$. Inserting the ansatz (1.2) into the system (1.1), Byeon et al.[6] got the following nonlocal semilinear elliptic equation

$$
-\Delta u+\omega u+\left(\frac{h^{2}(|x|)}{|x|^{2}}+\int_{|x|}^{\infty} \frac{h(s)}{s} u^{2}(s) d s\right) u=\lambda|u|^{p-2} u \text { in } \mathbb{R}^{2},
$$

where

$$
h(s)=h_{u}(s)=\frac{1}{2} \int_{0}^{s} r u^{2}(r) d r=\frac{1}{4 \pi} \int_{B_{s}} u^{2}(x) d x .
$$

Mathematically, equation (1.3) is not a pointwise identity as the appearance of the Chern-Simons term

$$
\left(\frac{h^{2}(|x|)}{|x|^{2}}+\int_{|x|}^{\infty} \frac{h(s)}{s} u^{2}(s) d s\right) u .
$$

Hence problem (1.3) is called a nonlocal problem and is quite different from the usual semi-linear Schrödinger equation. From the variational point of view, the nonlocal term causes some mathematical difficulties that make the study of problem (1.3) more interesting.

Following [6], equation (1.3) possesses a variational structure, that is, the standing wave solutions are obtained as critical points of the energy functional associated to (1.3) defined by

$$
\mathcal{L}(u)=\frac{1}{2} \int_{\mathbb{R}^{2}}\left(|\nabla u|^{2}+\omega u^{2}\right) d x+\frac{1}{2} \int_{\mathbb{R}^{2}} \frac{u^{2}}{|x|^{2}}\left(\int_{0}^{|x|} \frac{s}{2} u^{2}(s) d s\right)^{2} d x-\frac{\lambda}{p} \int_{\mathbb{R}^{2}}|u|^{p} d x,
$$

$u \in H_{r}^{1}\left(\mathbb{R}^{2}\right)$, where $H_{r}^{1}\left(\mathbb{R}^{2}\right):=\left\{u \in H^{1}\left(\mathbb{R}^{2}\right) \mid u(x)=u(|x|)\right\}$. In $[6,10,20,29,30,33,39,40,45]$, the critical points of $\mathcal{L}$ are found by using variational methods. It is shown that the value $p=4$ is critical for this problem. Indeed, for $p>4$, it is known that the energy functional is unbounded from below and satisfies a mountainpass geometry. In a certain sense, in this case the local nonlinearity dominates the nonlocal term. However the existence of a solution is not so direct, since for $p \in(4,6)$ the (PS)-condition is not known to hold. In the spirit of [34], this problem is bypassed in [6] by using a constrained minimization taking into account the Nehari-Pohozaev manifold.

A special case is $p=4$ : in this case, solutions have been explicitly found in $[6,7]$ as optimizers of a certain inequality. An alternative approach would be to pass to a self-dual equation, which leads to a Liouville equation that can be solved explicitly. For more information on the self-dual equations, see [14, 24]. For the 
case $p \geq 4$, [29] and [30] proved the existence, multiplicity, quantitative property and asymptotic behavior of normalized solutions with prescribed $L^{2}$-norm.

The situation is different if $p \in(2,4)$, solutions are found in [6] as minimizers on a $L^{2}$-sphere. Later, the results has been extended by Pomponio and Ruiz [32] by investigating the geometry of the energy functional under the different range of frequency $\omega$. Moreover, Pomponio and Ruiz in [33] also studied the bounded domain case for $p \in(2,4)$. By using singular perturbation arguments based on a Lyapunov-Schmidt reduction, they obtained some results on boundary concentration of solutions. Wan and Tan [40] studied the existence and multiplicity of standing waves for asymptotically linear nonlinearity case, and see [44] for the sublinear case. Cunha et al.[10] obtained a multiplicity result when the nonlinearity satisfies the general hypotheses introduced by Berestycki and Lions [8]. For more results about the initial value problem, well-posedness, existence and blow-up, scattering and uniqueness results for some nonlocal problems, we refer readers to $[5,11,12,19,21,26,27,42,43]$ and references therein.

When $p>6$, by using the symmetric mountain pass theorem, Huh [20] obtained the existence of infinitely many radially symmetric solutions for equation (1.3). Recently, this result has been extended to more general nonlinearity model by Zhang et al.[45]. Besides, Deng et al.[16] and Li et al.[31] investigated the existence and asymptotic behavior of radial sign-changing solutions by using constraint minimization method and quantitative deformation lemma for equation (1.3). Liu et al.[28] obtained a multiplicity result of sign-changing solutions via a novel perturbation approach and the method of invariant sets of descending flow.

Very recently, for the general 6-superlinear nonlinearity case, Tang et al.[39] considered the following nonlocal Schrödinger equation with the gauge field and deepening potential well

$$
-\Delta u+\lambda V(|x|) u+\left(\frac{h^{2}(|x|)}{|x|^{2}}+\int_{|x|}^{\infty} \frac{h(s)}{s} u^{2}(s) d s\right) u=f(u) \text { in } \mathbb{R}^{2},
$$

where the potential $V$ is a continuous function satisfies

$\left(V_{1}^{\prime}\right) V(|x|) \in C\left(\mathbb{R}^{2}\right)$ and $V(|x|) \geq 0$ on $\mathbb{R}^{2}$;

$\left(V_{2}\right)$ there exists a constant $b>0$ such that the set $V_{b}:=\left\{x \in \mathbb{R}^{2} \mid V(|x|)<b\right\}$ is nonempty and has finite measure;

$\left(V_{3}\right)$ there is bounded symmetric domain $\Omega$ such that $\Omega=\operatorname{int} V^{-1}(0)$ with smooth boundary $\partial \Omega$ and $\bar{\Omega}=$ $V^{-1}(0)$.

Under some suitable conditions on the nonlinearity $f$, the second and third authors proved the existence and multiplicity of solutions (possibly positive, negative or sign-changing) by using mountain pass theorem. Moreover, the concentration behavior of these solutions on the set $\Omega$ as $\lambda \rightarrow+\infty$ are also studied.

Involving the Chern-Simons-Schrödinger equations with potential wells, there is only the work [39] so far. As described above, the shape of solutions obtained in [39] may be single-bump. However, nothing is known for the existence of multi-bump type solutions. Motivated by the above facts, we intend in the present paper to study the existence of sign-changing multi-bump solutions for equation (1.5) with deepening potential well. To the best of our knowledge, it seems that such a problem was not considered in literature before. In order to state our statements, for the potential $V$ we need to assume that the following conditions besides $\left(V_{2}\right)$ and $\left(V_{3}\right)$,

$\left(V_{1}\right) V \in C^{1}\left(\mathbb{R}^{2}\right)$ and $V(|x|) \geq 0$ on $\mathbb{R}^{2} ;$

$\left(V_{4}\right)$ there are $k+1$ disjoint open bounded components $\Omega_{0}, \Omega_{1}, \Omega_{2}, \cdots, \Omega_{k}(k \geq 2)$ such that $\Omega=\operatorname{int} V^{-1}(0)=$ $\cup_{i=0}^{k} \Omega_{i}$ and $\operatorname{dist}\left(\Omega_{i}, \Omega_{j}\right)>0$ for $i \neq j, i, j=0,1,2, \cdots, k$, where $\Omega_{0}=\left\{x \in \mathbb{R}^{2}, r_{0}=0 \leq|x| \leq r_{0}^{\prime}\right\}$, $\Omega_{i}=\left\{x \in \mathbb{R}^{2}, r_{i} \leq|x| \leq r_{i}^{\prime}\right\}$.

Moreover, we suppose that the nonlinearity $f$ satisfies

$\left(f_{1}\right) f \in C(\mathbb{R}, \mathbb{R})$, and there exist constants $C>0$ and $q_{0} \in(4,+\infty)$ such that

$$
|f(t)| \leq C\left(|t|+|t|^{q_{0}-1}\right)
$$

$\left(f_{2}\right) f(t)=o(t)$ as $t \rightarrow 0$ 
$\left(f_{3}\right) \lim _{t \rightarrow \infty} \frac{F(t)}{t^{6}}=+\infty$ where $F(t)=\int_{0}^{t} f(s) d s$;

$\left(f_{4}\right)$ the function $\frac{f(t)}{t^{5}}$ is increasing on $(0,+\infty)$ and decreasing on $(-\infty, 0)$.

Before stating our results we first need to introduce some notations. Throughout this paper, we define

$$
H_{\lambda}:=\left\{u \in H_{r}^{1}\left(\mathbb{R}^{2}\right) \mid \int_{\mathbb{R}^{2}} \lambda V(|x|) u^{2} d x<+\infty\right\}
$$

with the norm

$$
\|u\|_{\lambda}^{2}=\int_{\mathbb{R}^{2}}|\nabla u|^{2}+\lambda V(|x|) u^{2} d x .
$$

Clearly, the embedding $H_{\lambda} \hookrightarrow H_{r}^{1}\left(\mathbb{R}^{2}\right)$ is continuous due to $\left(V_{1}\right)$ and $\left(V_{2}\right)$. We will give the proof later. Define the energy functional $\mathcal{J}_{\lambda}: H_{\lambda} \rightarrow \mathbb{R}$ by

$$
\mathcal{J}_{\lambda}(u)=\frac{1}{2} \int_{\mathbb{R}^{2}}\left(|\nabla u|^{2}+\lambda V(|x|) u^{2}\right) d x+\frac{1}{2} \int_{\mathbb{R}^{2}} \frac{u^{2}}{|x|^{2}}\left(\int_{0}^{|x|} \frac{s}{2} u^{2}(s) d s\right)^{2} d x-\int_{\mathbb{R}^{2}} F(u) d x,
$$

Then, our hypotheses imply that the functional $\mathcal{J}_{\lambda} \in C^{1}\left(H_{\lambda}, \mathbb{R}\right)$, and for any $u, \varphi \in H_{\lambda}$, we have

$$
\begin{aligned}
\left\langle J_{\lambda}^{\prime}(u), \varphi\right\rangle=\int_{\mathbb{R}^{2}} \nabla u \nabla \varphi+\lambda V(|x|) u \varphi d x+\int_{\mathbb{R}^{2}} \frac{h^{2}(|x|)}{|x|^{2}} u \varphi d x \\
\quad+\int_{\mathbb{R}^{2}} \frac{u^{2}}{|x|^{2}}\left(\int_{0}^{|x|} \frac{s}{2} u^{2}(s) d s\right)\left(\int_{0}^{|x|} s u(s) \varphi(s) d s\right) d x-\int_{\mathbb{R}^{2}} f(u) \varphi d x .
\end{aligned}
$$

Obviously, critical points of $\mathcal{J}_{\lambda}$ are the weak solutions for equation (1.5). Furthermore, if $u \in H_{\lambda}$ is a solution of (1.5) and $u^{ \pm} \neq 0$, then $u$ is a sign-changing solution of (1.5), where

$$
u^{+}(x)=\max \{u(x), 0\} \text { and } u^{-}(x)=\min \{u(x), 0\} .
$$

Our main result can be stated as follows.

Theorem 1.1. Suppose that $\left(V_{1}\right)-\left(V_{4}\right)$ and $\left(f_{1}\right)-\left(f_{4}\right)$ hold. Then, for any non-empty subset $T \subset\{0,1,2,3, \ldots, k\}$ with

$$
T=T_{1} \cup T_{2} \cup T_{3} \text { and } T_{i} \cap T_{j}=\emptyset \text { for } i \neq j, i, j=1,2,3 \text {. }
$$

There exists a constant $\Lambda_{T}>0$ such that for $\lambda>\Lambda_{T}$, equation (1.5) has a sign-changing multi-bump solution $u_{\lambda}$, which possesses the following property: for any sequence $\left\{\lambda_{n}\right\}$ with $\lambda_{n} \rightarrow+\infty$ as $n \rightarrow \infty$, there is a subsequences $\left\{u_{\lambda_{n_{i}}}\right\}$ converges strongly to $u$ in $H_{r}^{1}\left(\mathbb{R}^{2}\right)$, where $u \in H_{r}^{1}\left(\mathbb{R}^{2}\right)$ is a nontrivial solution of the equation

$$
\left\{\begin{array}{l}
-\Delta u+\left(\frac{h^{2}(|x|)}{|x|^{2}}+\int_{|x|}^{r_{k}^{\prime}} \frac{h(s)}{s} u^{2}(s) d s\right) u=f(u), \quad x \in \Omega_{T}=\cup_{i \in T} \Omega_{i}, \\
u=0 \quad x \in \mathbb{R}^{2} \backslash \Omega_{T} .
\end{array}\right.
$$

Moreover, $\left.u\right|_{\Omega_{i}}$ is positive for $i \in T_{1},\left.u\right|_{\Omega_{i}}$ is negative for $i \in T_{2}$, and $\left.u\right|_{\Omega_{i}}$ changes sign exactly once for $i \in T_{3}$.

The motivation of the present paper arises from the study of the local Schrödinger equations with deepening potential well

$$
-\Delta u+(\lambda V(x)+a(x)) u=f(x, u) \text { in } \mathbb{R}^{N} .
$$

We remark that conditions $\left(V_{1}\right)-\left(V_{3}\right)$ have been first introduced by Bartsch, Pankov and Wang [9] in studying the Schrödinger equation (1.10). They obtained some results on the existence of multiple solutions, and also showed that the solutions concentrated at the bottom of the potential well as $\lambda \rightarrow+\infty$. The existence and 
characterization of the solutions for equation (1.10) were considered in $[1,2,15,35]$ under conditions $\left(V_{1}\right)$ $\left(V_{4}\right)$. For example, Ding and Tanaka [15] first constructed the existence of multi-bump positive solutions $u_{\lambda}$ for (1.10), and they also proved that, up to a subsequence, $u_{\lambda}$ converges strongly in $H^{1}\left(\mathbb{R}^{N}\right)$ to a function $u$, which satisfies $u=0$ outside $\Omega_{T}$ and $\left.u\right|_{\Omega_{i}}$ is the positive least energy solution to the equation

$$
-\Delta u+a(x) u=f(x, u), u \in H_{0}^{1}\left(\Omega_{i}\right) .
$$

Inspired by [15], Alves [1] and Sato and Tanaka [35] investigated the sign-changing multi-bump solutions to equation (1.10) independently. Later, Alves and Pereira [2] obtained a similar result for the critical growth case. We must point out that the equation (1.11) (called limit equation of (1.10)) plays an important role in the study of multi-bump solutions for equation (1.10). Because the positive, negative and sign-changing solutions of (1.11) are used as building bricks to construct the multi-bump solutions of (1.10) by using of gluing techniques. Recently, there are some works focused on study of multi-bump solutions and ground states solutions for other nonlocal problems. For instance, see [18, 36, 37] for Schrödinger-Kirchhoff equation, $[3,13,38]$ for Schrödinger-Poisson system, and [4] for Choquard equation and so on.

From the commentaries above, it is quite natural to ask if the results in $[1,35]$ still hold for the ChernSimons-Schrödinger equations. Unfortunately, we can not draw a similar conclusion in a straight way. Since problem (1.5) is a nonlocal one as the appearance of the Chern-Simons term, then the solutions of problem (1.5) need their global information. Thus, we cannot use the same arguments explored in $[1,35]$ to solve the corresponding limit equation (1.9) separately on each $\Omega_{i}$. This result in the effective methods used [1, 35] for local Schrödinger equations cannot be applied to nonlocal problem (1.5) directly, there arises a technical problem one should overcome. As we all know, in order to obtain the existence of sign-changing multi-bump solutions for problem (1.5), we need to use the existence and some properties of the least energy sign-changing solutions of limit equation (1.9). However, it is not easy to prove the existence and sign properties of the least energy solution for limit equation (1.9). Hence, the first step is to consider the limit equation (1.9) and to look for the existence of least energy sign-changing solution that is nonzero on each component $\Omega_{i}, i \in T$.

Our result on the limit equation (1.9) can be stated as follows.

Theorem 1.2. Suppose that $\left(f_{1}\right)-\left(f_{4}\right)$ hold, then, for any non-empty subset $T$, equation (1.9) has a nontrivial solution $u$ with $\left.u\right|_{\Omega_{i}}$ is positive for $i \in T_{1},\left.u\right|_{\Omega_{i}}$ is negative for $i \in T_{2}$, and $\left.u\right|_{\Omega_{i}}$ changes sign exactly once for $i \in T_{3}$. Moreover, $u$ is the least energy solution among all solutions with those sign properties.

To prove our results, some arguments are in order. First, to obtain the least energy sign-changing solution solutions with prescribed sign properties for limit equation (1.9), we first prove the set $\mathcal{M}_{T}$ (see (2.1)) is nonempty and then we seek minimizers of the energy functional on $\mathcal{M}_{T}$. Observe that $\mathcal{M}_{T}$ is not a $C^{1}$. manifold, we will take advantage of constraint minimization method and quantitative deformation lemma to obtain the existence of minimizers of the energy functional. Second, we will use the penalization technique explored by del Pino and Felmer in [17] to cut off the nonlinearity $f$, then, to control the order of growth of nonlinearity $f$ outside the potential well $\Omega_{T}$. In such a way, we build a modification of the energy functional associated to (1.5) and give some energy relations of problems (1.5) and (1.9) which play a key role in getting the critical point of (1.5) (see Lemma 4.3). Moreover, in order to show a critical point associated to the modified functional is indeed a solution to the original problem, we also need give a delicate $L^{\infty}$-estimation for the solutions of the modified problem. Finally, we study a special minimax value of the modified functional, which is crucial for proving Theorem 1.1. Furthermore, via a rather precise analysis of deformation flow to the modified functional, we prove the existence of sign-changing multi-bump solutions for (1.5).

The paper is organized as follows. In Section 2, we give some preliminary lemmas and the proof of Theorem 1.2. In Section 3, we define a penalization problem and modified functional, and give a $L^{\infty}$-estimation for the solutions of the modified problem. In Section 4, we study a special minimax value of the modified functional. At last, we give the proof of Theorem 1.1 in Section 5.

Throughout the sequel, we denote the usual Lebesgue space with norms $\|u\|_{p}=\left(\int_{\mathbb{R}^{2}}|u|^{p} d x\right)^{\frac{1}{p}}$ by $L^{p}\left(\mathbb{R}^{2}\right)$, where $1 \leq p<\infty$, and $C$ denotes different positive constant in different place. 


\section{Mixed type sign-changing solutions to limit problem}

In this section, we study the existence of solutions for the limited equation (1.9) with prescribed sign properties. Firstly, we restrict the nonlinearity $\tilde{f}(x, t)=0$ if

$$
\text { (i) } t<0 \text { and } x \in \Omega_{i} \text { for } i \in T_{1} \text {; or (ii) } t>0 \text { and } x \in \Omega_{i} \text { for } i \in T_{2} \text {. }
$$

Denoted

with the norm

$$
H_{T}=\left\{u \in H_{r}^{1}\left(\mathbb{R}^{2}\right) \mid u=0 \text { in } x \in \mathbb{R}^{2} \backslash \Omega_{T}\right\}
$$

$$
\|u\|_{T}^{2}=\int_{\Omega_{T}}|\nabla u|^{2} d x .
$$

Now we define the energy functional $J_{T}$ corresponding to limit problem (1.9) on $H_{T}$

$$
J_{T}(u):=\frac{1}{2} \int_{\Omega_{T}}|\nabla u|^{2} d x+\frac{1}{2} \int_{\Omega_{T}} \frac{u^{2}}{|x|^{2}}\left(\int_{0}^{|x|} \frac{s}{2} u^{2}(s) d s\right)^{2} d x-\int_{\Omega_{T}} \tilde{F}(x, u) d x, u \in H_{T},
$$

and the set

$$
\begin{aligned}
& \mathcal{M}_{T}=\left\{u \in H_{T} \mid\left\langle J_{T}^{\prime}(u), u_{i}\right\rangle=0 \text { for } i \in T, u_{i}^{+} \neq 0 \text { for } i \in T_{1},\right. \\
&\left.u_{i}^{-} \neq 0 \text { for } i \in T_{2} \text { and }\left\langle J_{T}^{\prime}(u), u_{i}^{ \pm}\right\rangle=0, u_{i}^{ \pm} \neq 0 \text { for } i \in T_{3}\right\},
\end{aligned}
$$

where $u_{i}:=\left.u\right|_{\Omega_{i}}$ and $T=T_{1} \cup T_{2} \cup T_{3} \subset\{0,1,2,3, \ldots, k\}$ satisfies (1.8), and $\tilde{F}(x, u)=\int_{0}^{u} \tilde{f}(x, t) d t$. Let

$$
m=\inf _{u \in \mathcal{M}_{T}} J_{T}(u) .
$$

If $m$ is attained by $u_{0} \in \mathcal{M}_{T}$ and $J_{T}^{\prime}\left(u_{0}\right)=0$, then $u_{0}$ be called a the least energy sign-changing solution of limit problem (1.9).

Without loss of generality, we consider the case $T_{1}=\{1\}, T_{2}=\{2\}$ and $T_{3}=\{3\}$ for simplify. In this case, $\Omega_{T}=\cup_{i=1}^{3} \Omega_{i}$ with $\operatorname{dist}\left(\Omega_{i}, \Omega_{j}\right)>0$ for $i \neq j, i, j=1,2,3$. To simplify the notations, we use $\Omega, \mathcal{M}, H$ to denote the sets $\Omega_{T}, \mathcal{M}_{T}, H_{T}$ respectively. Moreover, we define the functional on $H$ as follows

$$
J(u):=\frac{1}{2} \int_{\Omega}|\nabla u|^{2} d x+\frac{1}{2} \int_{\Omega} \frac{u^{2}}{|x|^{2}}\left(\int_{0}^{|x|} \frac{s}{2} u^{2}(s) d s\right)^{2} d x-\int_{\Omega} \tilde{F}(x, u) d x, u \in H .
$$

The functional $J$ is well-defined and belongs to $C^{1}(H, \mathbb{R})$. Moreover, for any $u, \varphi \in H$, we have

$$
\begin{aligned}
\left\langle J^{\prime}(u), \varphi\right\rangle= & \int_{\Omega} \nabla u \nabla \varphi d x+\int_{\Omega} \frac{h^{2}(|x|)}{|x|^{2}} u \varphi d x+\int_{\Omega} \frac{u^{2}}{|x|^{2}}\left(\int_{0}^{|x|} \frac{s}{2} u^{2}(s) d s\right)\left(\int_{0}^{|x|} s u(s) \varphi(s) d s\right) d x \\
& -\int_{\Omega} \tilde{f}(x, u) \varphi d x .
\end{aligned}
$$

Clearly, critical points of $J$ are the weak solutions for limit problem (1.9).

We use constraint minimizer on $\mathcal{M}$ to seek a critical point of $J$ with nonzero component. We first check that the set $\mathcal{M}$ is nonempty in $H$.

Lemma 2.1. Assume that $\left(f_{1}\right)-\left(f_{4}\right)$ hold. For $u \in H$ with $u_{1}^{+} \neq 0, u_{2}^{-} \neq 0$ and $u_{3}^{ \pm} \neq 0$, then there exists a unique 4-tuple $\left(s_{1}, s_{2}, s_{3}, s_{4}\right) \in\left(\mathbb{R}_{+}\right)^{4}$ such that

$$
s_{1} u_{1}+s_{2} u_{2}+s_{3} u_{3}^{+}+s_{4} u_{3}^{-} \in \mathcal{M} .
$$

Proof. For $u \in H$ with $u_{1}^{+} \neq 0, u_{2}^{-} \neq 0$ and $u_{3}^{ \pm} \neq 0$, we define

$$
F(u)=\left(F_{1}(u), F_{2}(u), F_{3}(u), F_{4}(u)\right),
$$


where

$$
F_{i}(u)= \begin{cases}J^{\prime}(u) u_{i}, & i=1,2, \\ J^{\prime}(u)\left(u_{3}^{+}\right), & i=3, \\ J^{\prime}(u)\left(u_{3}^{-}\right), & i=4 .\end{cases}
$$

Obviously, $t_{1} u_{1}+t_{2} u_{2}+t_{3} u_{3}^{+}+t_{4} u_{3}^{-} \in \mathcal{N}$ if and only if

$$
F\left(t_{1} u_{1}+t_{2} u_{2}+t_{3} u_{3}^{+}+t_{4} u_{3}^{-}\right)=0 .
$$

Next, we obtain the desired results by proving two claims.

Claim 1. For $u \in H$ with $u_{1} \neq 0, u_{2} \neq 0$ and $u_{3}^{ \pm} \neq 0$, there exists at least one solution for (2.5).

Firstly, there exists a unique $\bar{t}_{1}>0$ such that

$$
F_{1}\left(\bar{t}_{1} u_{1}+t_{2} u_{2}+t_{3} u_{3}^{+}+t_{4} u_{3}^{-}\right)=0 .
$$

for fixed $\left(t_{2}, t_{3}, t_{4}\right) \in\left(\mathbb{R}_{+}\right)^{3}$. In fact, we define

$$
\begin{aligned}
& k(t):=t^{2} \int_{\Omega}\left|\nabla u_{1}\right|^{2} d x+\int_{\Omega}\left(\int_{0}^{|x|} \frac{s}{2}\left(t^{2} u_{1}^{2}(s)+t_{2}^{2} u_{2}^{2}(s)+t_{3}^{2} u_{3}^{+2}(s)+t_{4}^{2} u_{4}^{+2}(s)\right) d s\right)^{2} \frac{t^{2} u_{1}^{2}}{|x|^{2}} d x \\
& +\int_{\Omega} \frac{t^{2} u_{1}^{2}+t_{2}^{2} u_{2}^{2}+t_{3}^{2} u_{3}^{+2}+t_{4}^{2} u_{4}^{+2}}{|x|^{2}}\left(\int_{0}^{|x|} \frac{s}{2}\left(t^{2} u_{1}^{2}(s)+t_{2}^{2} u_{2}^{2}(s)+t_{3}^{2} u_{3}^{+2}(s)+t_{4}^{2} u_{4}^{+2}(s)\right) d s\right) \\
& \left(\int_{0}^{|x|} s t^{2} u_{1}^{2}(s) d s\right) d x-\int_{\Omega} \tilde{f}\left(x, t u_{1}\right) t u_{1} d x .
\end{aligned}
$$

By $\left(f_{2}\right),\left(f_{3}\right)$ and $\left(f_{4}\right)$, it is easy to see that $k(t)>0$ for $t>0$ small enough and $k(t)<0$ for $t>0$ large enough. Hence, there exists $\bar{t}_{1}>0$ such that $k\left(\bar{t}_{1}\right)=0$. Moreover, by $\left(f_{4}\right)$ we can deduce that $\bar{t}_{1}$ is unique. Thus, we can get a function $\eta_{1}:\left(\mathbb{R}_{+}\right)^{3} \rightarrow(0,+\infty)$ defined by

$$
\eta_{1}\left(t_{2}, t_{3}, t_{4}\right)=\bar{t}_{1},
$$

such that $F_{1}\left(\bar{t}_{1} u_{1}+t_{2} u_{2}+t_{3} u_{3}^{+}+t_{4} u_{3}^{-}\right)=0$.

By the same arguments as above, we can define function $\eta_{i}:\left(\mathbb{R}_{+}\right)^{3} \rightarrow(0,+\infty), i=2,3,4$, given by

$$
\eta_{2}\left(t_{1}, t_{3}, t_{4}\right)=\bar{t}_{2}, \quad \eta_{3}\left(t_{1}, t_{2}, t_{4}\right)=\bar{t}_{3}, \quad \eta_{4}\left(t_{1}, t_{2}, t_{3}\right)=\bar{t}_{4},
$$

satisfying $F_{2}\left(t_{1} u_{1}+\bar{t}_{2} u_{2}+t_{3} u_{3}^{+}+t_{4} u_{3}^{-}\right)=0, F_{3}\left(t_{1} u_{1}+t_{2} u_{2}+\bar{t}_{3} u_{3}^{+}+t_{4} u_{3}^{-}\right)=0$, and $F_{4}\left(t_{1} u_{1}+t_{2} u_{2}+t_{3} u_{3}^{+}+\bar{t}_{4} u_{3}^{-}\right)=0$.

Moreover, the functions $\eta_{i}:\left(\mathbb{R}_{+}\right)^{3} \rightarrow(0,+\infty), i=1,2,3,4$, have the following three properties:

(i) For any $\left(a_{1}, a_{2}, a_{3}, a_{4}\right) \in\left(\mathbb{R}_{+}\right)^{4}$, we have $\eta_{i}\left(a_{i} \mid a_{1}, a_{2}, a_{3}, a_{4}\right)>0$;

(ii) $\eta_{i}$ are continuous on $[0,+\infty)^{3}$;

(iii) If $a_{\max }^{i}$ large enough, then

$$
\bar{a}_{i}=\eta_{i}\left(a_{i} \mid a_{1}, a_{2}, a_{3}, a_{4}\right)<a_{\max }^{i}:=\max \left\{a_{1}, \ldots, a_{i-1}, a_{i+1}, \ldots, a_{4}\right\}
$$

where $\left(a_{i} \mid a_{1}, a_{2}, a_{3}, a_{4}\right):=\left(a_{1}, \ldots, a_{i-1}, a_{i+1}, \ldots, a_{4}\right)$.

By the property (iii), there exists $M_{1}>0$ such that $\eta_{i}\left(a_{i} \mid a_{1}, a_{2}, a_{3}, a_{4}\right) \leq a_{\max }^{i}$ for $a_{\max }^{i}>M_{1}$. From the property $(i)$, we get

$$
M_{2}:=\max _{i}\left\{\max \eta_{i}\left(a_{i} \mid a_{1}, a_{2}, a_{3}, a_{4}\right): a_{\max }^{i} \leq M_{1}, i=1,2,3,4\right\}>0 .
$$

Thus, $M_{0}:=\max \left\{M_{1}, M_{2}\right\}>0$. For any $\left(a_{1}, a_{2}, a_{3}, a_{4}\right) \in\left[0, M_{0}\right]^{4}$, it follows from (iii) that $\eta_{i}\left(a_{i} \mid a_{1}, a_{2}, a_{3}, a_{4}\right) \leq M_{0}$. 
Hence, we can define $L:\left[0, M_{0}\right]^{4} \rightarrow\left[0, M_{0}\right]^{4}$ by

$$
L\left(a_{1}, a_{2}, a_{3}, a_{4}\right):=\left(\bar{a}_{1}, \bar{a}_{2}, \bar{a}_{3}, \bar{a}_{4}\right),
$$

where $\bar{a}_{i}=\eta_{i}\left(a_{i} \mid a_{1}, a_{2}, a_{3}, a_{4}\right)$ satisfying $F_{1}\left(\bar{a}_{1} u_{1}+a_{2} u_{2}+a_{3} u_{3}^{+}+a_{4} u_{3}^{-}\right)=0, F_{2}\left(a_{1} u_{1}+\bar{a}_{2} u_{2}+a_{3} u_{3}^{+}+a_{4} u_{3}^{-}\right)=0$, $F_{3}\left(a_{1} u_{1}+a_{2} u_{2}+\bar{a}_{3} u_{3}^{+}+a_{4} u_{3}^{-}\right)=0, F_{4}\left(a_{1} u_{1}+a_{2} u_{2}+a_{3} u_{3}^{+}+\bar{a}_{4} u_{3}^{-}\right)=0$.

Obviously, $L\left(a_{1}, a_{2}, a_{3}, a_{4}\right)$ is continuous on $\left[0, M_{0}\right]^{4}$. Now, by applying the Brouwer Fixed Point Theorem, there exists $\left(s_{1}, s_{2}, s_{3}, s_{4}\right) \in\left[0, M_{0}\right]^{4}$ such that

$$
L\left(s_{1}, s_{2}, s_{3}, s_{4}\right)=\left(s_{1}, s_{2}, s_{3}, s_{4}\right) .
$$

Thus, $\left(s_{1}, s_{2}, s_{3}, s_{4}\right)$ is a solution of (2.5).

Claim 2. $\left(s_{1}, s_{2}, s_{3}, s_{4}\right)$ obtained by Claim 1 is the unique solution of (2.5). To show Claim 2, the proof will be carried out in two cases.

Case 1. $u \in \mathcal{M}$. In this case, the 4-tuple $(1,1,1,1)$ is a solution of $(2.5)$. We prove that $(1,1,1,1)$ is the unique solution of $(2.5)$ in $\left(\mathbb{R}^{+}\right)^{4}$. Indeed, suppose $\left(a_{1}, a_{2}, a_{3}, a_{4}\right)$ be any other solution, then

$$
F_{i}\left(a_{1} u_{1}+a_{2} u_{2}+a_{3} u_{3}^{+}+a_{4} u_{3}^{-}\right)=0, i=1,2,3,4 .
$$

Without loss of generality, we suppose $a_{1}=\max \left\{a_{1}, a_{2}, a_{3}, a_{4}\right\}$. That is

$$
\begin{aligned}
& a_{1}^{2} \int_{\Omega}\left|\nabla u_{1}\right|^{2} d x+\int_{\Omega}\left(\int_{0}^{|x|} \frac{s}{2}\left(a_{1}^{2} u_{1}^{2}(s)+a_{2}^{2} u_{2}^{2}(s)+a_{3}^{2} u_{3}^{+2}(s)+a_{4}^{2} u_{4}^{+2}(s)\right) d s\right)^{2} \frac{a_{1}^{2} u_{1}^{2}}{|x|^{2}} d x \\
& +\int_{\Omega} \frac{a_{1}^{2} u_{1}^{2}+a_{2}^{2} u_{2}^{2}+a_{3}^{2} u_{3}^{+2}+a_{4}^{2} u_{4}^{+2}}{|x|^{2}}\left(\int_{0}^{|x|} \frac{s}{2}\left(a_{1}^{2} u_{1}^{2}(s)+a_{2}^{2} u_{2}^{2}(s)+a_{3}^{2} u_{3}^{+2}(s)+a_{4}^{2} u_{4}^{+2}(s)\right) d s\right) \\
& \left(\int_{0}^{|x|} s a_{1}^{2} u_{1}^{2}(s) d s\right) d x=\int_{\Omega} \tilde{f}\left(x, a_{1} u_{1}\right) a_{1} u_{1} d x .
\end{aligned}
$$

Since $u \in \mathcal{M}$, we have

$$
\begin{aligned}
& \int_{\Omega}\left|\nabla u_{1}\right|^{2} d x+\int_{\Omega}\left(\int_{0}^{|x|} \frac{s}{2}\left(u_{1}^{2}(s)+u_{2}^{2}(s)+u_{3}^{+2}(s)+u_{4}^{+2}(s)\right) d s\right)^{2} \frac{u_{1}^{2}}{|x|^{2}} d x \\
& +\int_{\Omega} \frac{u_{1}^{2}+u_{2}^{2}+u_{3}^{+2}+u_{4}^{+2}}{|x|^{2}}\left(\int_{0}^{|x|} \frac{s}{2}\left(u_{1}^{2}(s)+u_{2}^{2}(s)+u_{3}^{+2}(s)+u_{4}^{+2}(s)\right) d s\right) \\
& \left(\int_{0}^{|x|} s u_{1}^{2}(s) d s\right) d x=\int_{\Omega} \tilde{f}\left(x, u_{1}\right) u_{1} d x .
\end{aligned}
$$

So we obtain

$$
\left(\frac{1}{a_{1}^{4}}-1\right) \int_{\Omega}\left|\nabla u_{1}\right|^{2} d x \geq \int_{\Omega}\left(\frac{\tilde{f}\left(x, a_{1} u_{1}\right)}{\left(a_{1} u_{1}\right)^{5}}-\frac{\tilde{f}\left(x, u_{1}\right)}{\left(u_{1}\right)^{5}}\right) u_{1}^{6} d x .
$$

By $\left(f_{1}\right)-\left(f_{4}\right)$, it imply that $a_{1}=\max \left\{a_{1}, a_{2}, a_{3}, a_{4}\right\} \leq 1$. By the same arguments, we can easily conclude that $\min \left\{a_{1}, a_{2}, a_{3}, a_{4}\right\} \geq 1$. Consequently, the 4-tuple $(1,1,1,1)$ is the unique solution of (2.5).

Case 2. $u \notin \mathcal{M}$. If $\left(u_{1}, u_{2}, u_{3}, u_{4}\right) \notin \mathcal{N}$, then by Claim 1 , we know that $(2.5)$ has a solution $\left(s_{1}, s_{2}, s_{3}, s_{4}\right)$. Assume that $\left(s_{1}^{\prime}, s_{2}^{\prime}, s_{3}^{\prime}, s_{4}^{\prime}\right)$ also be a solution. Then we have

$$
\frac{s_{1}^{\prime}}{s_{1}} s_{1} u_{1}+\frac{s_{2}^{\prime}}{s_{2}} s_{2} u_{2}+\frac{s_{3}^{\prime}}{s_{3}} s_{3} u_{3}^{+}+\frac{s_{4}^{\prime}}{s_{4}} s_{4} u_{3}^{-} \in \mathcal{M} .
$$


Since $s_{1} u_{1}+s_{2} u_{2}+s_{3} u_{3}^{+}+s_{4} u_{3}^{-} \in \mathcal{M}$, by Case 1 we get

$$
\frac{s_{1}^{\prime}}{s_{1}}=\frac{s_{2}^{\prime}}{s_{2}}=\frac{s_{3}^{\prime}}{s_{3}}=\frac{s_{4}^{\prime}}{s_{4}}=1 .
$$

Thus, $\left(s_{1}, s_{2}, s_{3}, s_{4}\right)$ is the unique solution of $(2.5)$ in $\left(\mathbb{R}_{+}\right)^{4}$.

Lemma 2.2. Assume that $\left(f_{1}\right)-\left(f_{4}\right)$ hold, then the unique 4-tuple $\left(t_{1}, t_{2}, t_{3}, t_{4}\right) \in\left(\mathbb{R}_{+}\right)^{4}$ obtained by Lemma 2.1 is the unique maximum point of the function $\varphi:\left(\mathbb{R}_{+}\right)^{4} \rightarrow \mathbb{R}$ defined by

$$
\varphi\left(s_{1}, s_{2}, s_{3}, s_{4}\right)=J\left(s_{1} u_{1}+s_{2} u_{2}+s_{3} u_{3}^{+}+s_{4} u_{3}^{-}\right) .
$$

Proof. From the proof of Lemma 2.1, we know that $\left(t_{1}, t_{2}, t_{3}, t_{4}\right)$ is the unique critical point of $\varphi$ in $\left(\mathbb{R}_{+}\right)^{4}$. By the assumption $\left(f_{3}\right)$, we deduce that $\varphi\left(t_{1}, t_{2}, t_{3}, t_{4}\right) \rightarrow-\infty$ as $\left|\left(t_{1}, t_{2}, t_{3}, t_{4}\right)\right| \rightarrow \infty$, so it is sufficient to check that a maximum point cannot be achieved on the boundary of $\left(\mathbb{R}_{+}\right)^{4}$. Choose $\left(s_{1}, s_{2}, s_{3}, s_{4}\right) \in \partial\left(\mathbb{R}_{+}\right)^{4}$, without loss of generality, we may assume that $s_{1}=0$. But, it is obviously that

$$
\begin{aligned}
l(s) & =\varphi\left(s, s_{2}, s_{3}, s_{4}\right)=J\left(s u_{1}+s_{2} u_{2}+s_{3} u_{3}^{+}+s_{4} u_{3}^{-}\right) \\
& =\frac{1}{2} \int_{\Omega}\left|\nabla\left(s u_{1}+s_{2} u_{2}+s_{3} u_{3}^{+}+s_{4} u_{3}^{-}\right)\right|^{2} d x \\
& +\frac{1}{2} \int_{\Omega} \frac{\left(s u_{1}+s_{2} u_{2}+s_{3} u_{3}^{+}+s_{4} u_{3}^{-}\right)^{2}}{|x|^{2}}\left(\int_{0}^{|x|} \frac{s}{2}\left(s u_{1}(s)+s_{2} u_{2}(s)+s_{3} u_{3}^{+}(s)+s_{4} u_{3}^{-}(s)\right)^{2} d s\right)^{2} d x \\
& -\int_{\Omega} \tilde{F}\left(x, s u_{1}+s_{2} u_{2}+s_{3} u_{3}^{+}+s_{4} u_{3}^{-}\right) d x
\end{aligned}
$$

is an increasing function with respect to $s$ if $s>0$ is small enough, $\left(0, s_{2}, s_{3}, s_{4}\right)$ is impossible to be a maximum point of $\varphi$.

Lemma 2.3. Assume that $\left(f_{1}\right)-\left(f_{4}\right)$ hold, let $u \in H$ with $u_{1}^{+} \neq 0, u_{2}^{-} \neq 0$ and $u_{3}^{ \pm} \neq 0$ such that, $F_{i}(u) \leq 0$ for $i=1,2,3,4$, where $F_{i}$ are given by (2.4). Then the unique 4-tuples $\left(t_{1}, t_{2}, t_{3}, t_{4}\right) \in\left(\mathbb{R}_{+}\right)^{4}$ obtained by Lemma 2.1 satisfied $\left(t_{1}, t_{2}, t_{3}, t_{4}\right) \in(0,1]^{4}$.

Proof. Without loss of generality, we suppose that $t_{1}=\max \left\{t_{1}, t_{2}, t_{3}, t_{4}\right\}$. Since $t_{1} u_{1}+t_{2} u_{2}+t_{3} u_{3}^{+}+t_{4} u_{3}^{-} \in \mathcal{M}$, we get

$$
\begin{aligned}
& t_{1}^{2} \int_{\Omega}\left|\nabla u_{1}\right|^{2} d x+\int_{\Omega}\left(\int_{0}^{|x|} \frac{s}{2}\left(t_{1}^{2} u_{1}^{2}(s)+t_{2}^{2} u_{2}^{2}(s)+t_{3}^{2} u_{3}^{+2}(s)+t_{4}^{2} u_{4}^{+2}(s)\right) d s\right)^{2} \frac{t_{1}^{2} u_{1}^{2}}{|x|^{2}} d x \\
& +\int_{\Omega} \frac{t_{1}^{2} u_{1}^{2}+t_{2}^{2} u_{2}^{2}+t_{3}^{2} u_{3}^{+2}+t_{4}^{2} u_{4}^{+2}}{|x|^{2}}\left(\int_{0}^{|x|} \frac{s}{2}\left(t_{1}^{2} u_{1}^{2}(s)+t_{2}^{2} u_{2}^{2}(s)+t_{3}^{2} u_{3}^{+2}(s)+t_{4}^{2} u_{4}^{+2}(s)\right) d s\right) \\
& \left(\int_{0}^{|x|} s t_{1}^{2} u_{1}^{2}(s) d s\right) d x=\int_{\Omega} \tilde{f}\left(x, t_{1} u_{1}\right) t_{1} u_{1} d x .
\end{aligned}
$$

At the same time, using $F_{i}(u) \leq 0$, one has

$$
\begin{aligned}
& \int_{\Omega}\left|\nabla u_{1}\right|^{2} d x+\int_{\Omega}\left(\int_{0}^{|x|} \frac{s}{2}\left(u_{1}^{2}(s)+u_{2}^{2}(s)+u_{3}^{+2}(s)+u_{4}^{+2}(s)\right) d s\right)^{2} \frac{u_{1}^{2}}{|x|^{2}} d x \\
& +\int_{\Omega} \frac{u_{1}^{2}+u_{2}^{2}+u_{3}^{+2}+u_{4}^{+2}}{|x|^{2}}\left(\int_{0}^{|x|} \frac{s}{2}\left(u_{1}^{2}(s)+u_{2}^{2}(s)+u_{3}^{+2}(s)+u_{4}^{+2}(s)\right) d s\right) \\
& \left(\int_{0}^{|x|} s u_{1}^{2}(s) d s\right) d x \leq \int_{\Omega} \tilde{f}\left(x, u_{1}\right) u_{1} d x .
\end{aligned}
$$


Therefore, (2.7) and (2.8) imply that

$$
\left(\frac{1}{t_{1}^{4}}-1\right) \int_{\Omega}\left|\nabla u_{1}\right|^{2} d x \geq \int_{\Omega}\left(\frac{\tilde{f}\left(x, t_{1} u_{1}\right)}{t_{1}^{5} u_{1}^{5}}-\frac{\tilde{f}\left(x, u_{1}\right)}{u_{1}^{5}}\right) u_{1}^{6} d x .
$$

By $\left(f_{4}\right)$, we obtain $t_{1} \leq 1$. Thus we complete the proof.

Next, we consider the following constrained minimization problem $m:=\inf _{u \in \mathcal{M}} J(u)$.

Lemma 2.4. Assume that $\left(f_{1}\right)-\left(f_{4}\right)$ hold, then $m>0$ and $m$ can be achieved by a function $v \in \mathcal{M}$.

Proof. Let $u \in \mathcal{M}$, then

$$
\int_{\Omega}\left|\nabla u_{i}\right|^{2} d x \leq \int_{\Omega} \tilde{f}\left(x, u_{i}\right) u_{i} d x \text { for } i=1,2,
$$

and

$$
\int_{\Omega}\left|\nabla u_{3}^{ \pm}\right|^{2} d x \leq \int_{\Omega} \tilde{f}\left(x, u_{3}^{ \pm}\right) u_{3}^{ \pm} d x .
$$

Thus, by $\left(f_{1}\right),\left(f_{2}\right)$ and Sobolev embedding theorem, there exists a positive constant $C$ such that

$$
\left\|u_{1}^{+}\right\|,\left\|u_{2}^{-}\right\|,\left\|u_{3}^{+}\right\|,\left\|u_{3}^{-}\right\| \geq C .
$$

Therefore,

$$
\begin{aligned}
J(u) & =J(u)-\frac{1}{6} \sum_{i=1}^{3}\left\langle J^{\prime}(u), u_{i}\right\rangle=\sum_{i=1}^{3}\left(\frac{1}{3} \int_{\Omega}\left|\nabla u_{i}\right|^{2} d x+\int_{\Omega} \frac{1}{6} \tilde{f}\left(x, u_{i}\right) u_{i}-\tilde{F}\left(x, u_{i}\right) d x\right) \\
& \geq \frac{1}{3} \sum_{i=1}^{3} \int_{\Omega}\left|\nabla u_{i}\right|^{2} d x \geq C,
\end{aligned}
$$

this implies that $m>0$. Suppose that $\left\{v_{n}\right\} \subset \mathcal{M}$ satisfying

$$
\lim _{n \rightarrow+\infty} J\left(v_{n}\right)=m,
$$

we can easily to get that

$$
0<C_{1} \leq\left\|v_{n, i}\right\| \leq C_{2} \text { for } i=1,2 \text { and } C_{1} \leq\left\|v_{n, 3}^{ \pm}\right\| \leq C_{2} .
$$

Passing to a subsequences, one has

$$
\begin{aligned}
& v_{n} \rightarrow v \text { weakly in } H, \\
& v_{n} \rightarrow v \text { strongly in } L^{p}(\Omega) \text { for } 2 \leq p<\infty, \\
& v_{n} \rightarrow v \text { a.e. in } \Omega .
\end{aligned}
$$

Assumptions $\left(f_{1}\right)$ and $\left(f_{2}\right)$ give

$$
\int_{\Omega} \tilde{f}\left(x, v_{3}^{ \pm}\right) v_{3}^{ \pm} d x=\lim _{n \rightarrow+\infty} \int_{\Omega} \tilde{f}\left(x, v_{n, 3}^{ \pm}\right) v_{n, 3}^{ \pm} d x \geq \liminf _{n \rightarrow+\infty}\left\|v_{n, 3}^{ \pm}\right\|^{2} \geq C_{1} .
$$

Thus, $v_{3}^{ \pm} \neq 0$. By the same arguments, we conclude that $v_{1}^{+} \neq 0$ and $v_{2}^{-} \neq 0$. By Lemma 2.1, there exists a unique 4-tuple $\left(t_{1}, t_{2}, t_{3}, t_{4}\right) \in\left(\mathbb{R}_{+}\right)^{4}$ such that

$$
\bar{v}:=t_{1} v_{1}+t_{2} v_{2}+t_{3} v_{3}^{+}+t_{4} v_{3}^{-} \in \mathcal{M} .
$$

On the other hand, due to $\left\{v_{n}\right\} \subset \mathcal{M}$, we have that

$$
F_{i}(v) \leq 0 \text { for } i=1,2,3 .
$$

Thus, Lemma 2.3 deduces that

$$
\left(t_{1}, t_{2}, t_{3}, t_{4}\right) \in(0,1]^{4} .
$$


Thanks to the function $s f(s)-6 F(s)$ is a non-negative function, increasing on $(0,+\infty)$, decreasing on $(-\infty, 0)$, it follow from (2.10) and Lemma 2.2, we have

$$
\begin{aligned}
6 m \leq & 6 J(\bar{v})=6 J(\bar{v})-\sum_{i=1}^{2}\left\langle J^{\prime}(\bar{v}), t_{i} v_{i}\right\rangle-\left\langle J^{\prime}(\bar{v}), t_{3} v_{3}^{+}\right\rangle-\left\langle J^{\prime}(\bar{v}), t_{4} v_{4}^{-}\right\rangle \\
= & \sum_{i=1}^{2} 2 t_{i}^{2}\left\|v_{i}\right\|^{2}+2 t_{3}^{2}\left\|v_{3}^{+}\right\|^{2}+2 t_{4}^{2}\left\|v_{3}^{-}\right\|^{2}+\sum_{i=1}^{2} \int_{\Omega}\left(\tilde{f}\left(x, t_{i} v_{i}\right) t_{i} v_{i}-6 \tilde{F}\left(x, t_{i} v_{i}\right)\right) d x \\
& +\int_{\Omega}\left(\tilde{f}\left(x, t_{3} v_{3}^{+}\right) t_{3} v_{3}^{+}-6 \tilde{F}\left(x, t_{3} v_{3}^{+}\right)\right) d x+\int_{\Omega}\left(\tilde{f}\left(x, t_{4} v_{3}^{-}\right) t_{4} v_{3}^{-}-6 \tilde{F}\left(x, t_{4} v_{3}^{-}\right)\right) d x \\
\leq & \liminf _{n \rightarrow+\infty}\left\{\sum_{i=1}^{2} 2\left\|v_{n, i}\right\|^{2}+2\left\|v_{n, 3}^{+}\right\|^{2}+2\left\|v_{n, 3}^{-}\right\|^{2}+\sum_{i=1}^{2} \int_{\Omega}\left(\tilde{f}\left(x, v_{n, i}\right) v_{n, i}-6 \tilde{F}\left(x, v_{n, i}\right)\right) d x\right. \\
& \left.+\int_{\Omega}\left(\tilde{f}\left(x, v_{n, 3}^{+}\right) v_{n, 3}^{+}-6 \tilde{F}\left(x, v_{n, 3}^{+}\right)\right) d x+\int_{\Omega}\left(\tilde{f}\left(x, v_{n, 3}^{-}\right) v_{n, 3}^{-}-6 \tilde{F}\left(x, v_{n, 3}^{-}\right)\right) d x\right\} \\
\leq & \liminf _{n \rightarrow+\infty} 6 J\left(v_{n}\right)=6 m .
\end{aligned}
$$

Thus, $t_{1}=t_{2}=t_{3}=t_{4}=1$ and $m$ is attained by $v$. Since the restriction on $\tilde{f}(x, u)$, we can get that $v_{1} \geq 0$, $v_{2} \leq 0$ and $\left(v_{3}^{ \pm}\right) \neq 0$. So we prove the conclusion.

Proof of Theorem 1.2. We prove indirectly and assume that $\Phi^{\prime}(v) \neq 0$. Then there exist $\delta>0$ and $\varrho>0$ such that

$$
\|u-v\| \leq 3 \delta \Rightarrow\left\|\Phi^{\prime}(u)\right\|_{H^{*}} \geq \varrho .
$$

For convenient, we define the function

$$
g\left(s_{1}, s_{2}, s_{3}, s_{4}\right)=s_{1} v_{1}+s_{2} v_{2}+s_{3} v_{3}^{+}+s_{4} v_{3}^{-}, \quad\left(s_{1}, s_{2}, s_{3}, s_{4}\right) \in D=\left(\frac{1}{2}, \frac{3}{2}\right)^{4} .
$$

By Lemma 2.2, we have

$$
\bar{m}:=\max _{\left(s_{1}, s_{2}, s_{3}, s_{4}\right) \in \partial D} J\left(g\left(s_{1}, s_{2}, s_{3}, s_{4}\right)\right)<m .
$$

For $\varepsilon:=\min \{(m-\bar{m}) / 2, \varrho \delta / 8\}$ and $S:=B(v, \delta)$, then [41] yields a deformation $\eta \in C([0,1] \times H, H)$ such that

(i) $\eta(1, u)=u$ if $J(u)<m-2 \varepsilon$ or $J(u)>m+2 \varepsilon$;

(ii) $\eta\left(1, J^{m_{0}+\varepsilon} \cap B(v, \delta)\right) \subset J^{m-\varepsilon}$;

(iii) $J(\eta(1, u)) \leq J(u), \forall u \in H$;

(iv) $\eta(1, u)$ is a homeomorphism of $H$.

It is clear that

$$
\max _{\left(s_{1}, s_{2}, s_{3}, s_{4}\right) \in \bar{D}} J\left(\eta\left(1, g\left(s_{1}, s_{2}, s_{3}, s_{4}\right)\right)\right)<m .
$$

We claim that $\eta(1, g(D)) \cap \mathcal{M} \notin \emptyset$. In fact, define

$$
h\left(s_{1}, s_{2}, s_{3}, s_{4}\right):=\eta\left(1, g\left(s_{1}, s_{2}, s_{3}, s_{4}\right)\right)
$$

and

$$
\begin{aligned}
& \Psi_{0}\left(s_{1}, s_{2}, s_{3}, s_{4}\right):=\left(\frac{1}{s_{1}} F_{1} \circ g, \frac{1}{s_{2}} F_{2} \circ g, \frac{1}{s_{3}} F_{3} \circ g, \frac{1}{s_{4}} F_{4} \circ g\right)\left(s_{1}, s_{2}, s_{3} . s_{4}\right), \\
& \Psi_{1}\left(s_{1}, s_{2}, s_{3}, s_{4}\right):=\left(\frac{1}{s_{1}} F_{1} \circ h, \frac{1}{s_{2}} F_{2} \circ h, \frac{1}{s_{3}} F_{3} \circ h, \frac{1}{s_{4}} F_{4} \circ h\right)\left(s_{1}, s_{2}, s_{3} . s_{4}\right),
\end{aligned}
$$

Then the degree theory and Lemma 2.1 yield

$$
\operatorname{deg}\left(\Psi_{0}, D, 0\right)=1 .
$$

It follows from $(i)$, one has $g=h$ on $\partial D$. Thus, we obtain

$$
\operatorname{deg}\left(\Psi_{1}, D, 0\right)=\operatorname{deg}\left(\Psi_{0}, D, 0\right)=1
$$


Thus, $\Psi_{1}\left(\bar{s}_{1}, \bar{s}_{2}, \bar{s}_{3}, \bar{s}_{4}\right)=0$ for some $\left(\bar{s}_{1}, \bar{s}_{2}, \bar{s}_{3}, \bar{s}_{4}\right) \in D$, this implies that $\eta(1, g(D)) \cap \mathcal{M} \neq 0$.

By (2.13) and the definition of $m$ we reach a contradiction. Thus, $J^{\prime}(v)=0$. We can get $v \in C^{2}(\Omega)$ by standard elliptic regularity theory. So $v_{1}>0, v_{2}<0$ by the Maximum Principle. At last, we show that $v_{3}$ indeed has two nodal domains. Arguing by contradiction, we may assume that

$$
v_{3}=w_{1}+w_{2}+w_{3}, w_{1}, w_{2}, w_{3} \in H_{0}^{1}\left(\Omega_{3}\right),
$$

with $w_{i} \neq 0, w_{1} \geq 0, w_{2} \leq 0$ and $\operatorname{suppt}\left(w_{i}\right) \cap \operatorname{suppt}\left(w_{j}\right)=\emptyset$, for $i \neq j, i, j=1,2,3$ and

$$
\left\langle J^{\prime}(v), w_{i}\right\rangle=0 \text {, for } i=1,2,3 .
$$

Let $u:=w_{1}+w_{2}$, we see that $u^{+}=w_{1}$ and $u^{-}=w_{2}$. Setting

$$
\bar{v}:=v_{1}+v_{2}+u \in H_{0}^{1}(\Omega) .
$$

By Lemma 2.1, there exists a unique 4-tuple $\left(\bar{t}_{1}, \bar{t}_{2}, \bar{t}_{3}, \bar{t}_{4}\right) \in\left(\mathbb{R}_{+}\right)^{4}$ such that

$$
\bar{t}_{1} v_{1}+\bar{t}_{2} v_{2}+\bar{t}_{3} u^{+}+\bar{t}_{4} u^{-} \in \mathcal{M} \text {. }
$$

Therefore

$$
J\left(\bar{t}_{1} v_{1}+\bar{t}_{2} v_{2}+\bar{t}_{3} u^{+}+\bar{t}_{4} u^{-}\right) \geq m .
$$

Moreover, $J^{\prime}(v) w_{i}=0$ implies that $F_{i}(\bar{v})<0$ for $i=1$, 2, 3, 4. Using Lemma 2.3, we deduce that $\left(\bar{t}_{1}, \bar{t}_{2}, \bar{t}_{3}, \bar{t}_{4}\right) \in$ $(0,1]^{4}$. At the same time,

$$
\begin{aligned}
0 & =\frac{1}{6}\left\langle J^{\prime}(v), w_{3}\right\rangle=\frac{1}{6} \int_{\Omega}\left|\nabla w_{3}\right|^{2} d x \\
& +\frac{1}{6} \int_{\Omega}\left(\int_{0}^{|x|} \frac{s}{2}\left(v_{1}^{2}(s)+v_{2}^{2}(s)+w_{1}^{2}(s)+w_{2}^{2}(s)+w_{3}^{2}(s)\right) d s\right)^{2} \frac{w_{3}^{2}}{|x|^{2}} d x \\
& +\frac{1}{6} \int_{\Omega} \frac{v_{1}^{2}+v_{2}^{2}+w_{1}^{2}+w_{2}^{2}+w_{3}^{2}}{|x|^{2}}\left(\int _ { 0 } ^ { | x | } \frac { s } { 2 } \left(v_{1}^{2}(s)+v_{2}^{2}(s)+w_{1}^{2}(s)\right.\right. \\
& \left.\left.+w_{2}^{2}(s)+w_{3}^{2}(s)\right) d s\right)\left(\int_{0}^{|x|} s w_{3}^{2}(s) d s\right) d x-\frac{1}{6} \int \tilde{f}_{\Omega}\left(x, w_{3}\right) w_{3} d x \\
& <\frac{1}{2} \int_{\Omega}\left|\nabla w_{3}\right|^{2} d x+\frac{1}{6} \int_{\Omega}\left(\int_{0}^{|x|} \frac{s}{2}\left(v_{1}^{2}(s)+v_{2}^{2}(s)+w_{1}^{2}(s)+w_{2}^{2}(s)+w_{3}^{2}(s)\right) d s\right)^{2} \frac{w_{3}^{2}}{|x|^{2}} d x \\
& +\frac{1}{6} \int_{\Omega} \frac{v_{1}^{2}+v_{2}^{2}+w_{1}^{2}+w_{2}^{2}+w_{3}^{2}}{|x|^{2}}\left(\int _ { 0 } ^ { | x | } \frac { s } { 2 } \left(v_{1}^{2}(s)+v_{2}^{2}(s)+w_{1}^{2}(s)\right.\right. \\
& \left.\left.+w_{2}^{2}(s)+w_{3}^{2}(s)\right) d s\right)\left(\int_{0}^{|x|} s w_{3}^{2}(s) d s\right) d x-\int_{\Omega} \tilde{F}\left(x, w_{3}\right) d x
\end{aligned}
$$


and

$$
\begin{aligned}
& J\left(\bar{t}_{1} v_{1}+\bar{t}_{2} v_{2}+\bar{t}_{3} u^{+}+\bar{t}_{4} u^{-}\right) \\
& =J(\cdot)-\frac{1}{6}\left(\sum_{i=1}^{2}\left\langle J^{\prime}(\cdot), \bar{t}_{i} v_{i}\right\rangle+\left\langle J^{\prime}(\cdot), \bar{t}_{3} u_{3}^{+}\right\rangle+\left\langle J^{\prime}(\cdot), \bar{t}_{4} u_{4}^{-}\right\rangle\right) \\
& =\frac{1}{3} \sum_{i=1}^{2} \bar{t}_{i}^{2}\left\|v_{i}\right\|^{2}+\frac{1}{3} \bar{t}_{3}^{2}\left\|u_{3}^{+}\right\|^{2}+\frac{1}{3} \bar{t}_{4}^{2}\left\|u_{3}^{-}\right\|^{2}+\frac{1}{6} \sum_{i=1}^{2} \int_{\Omega}\left(\tilde{f}\left(x, t_{i} v_{i}\right) t_{i} v_{i}-6 \tilde{F}\left(x, t_{i} v_{i}\right)\right) d x \\
& +\frac{1}{6} \int_{\Omega}\left(\tilde{f}\left(x, t_{3} u_{3}^{+}\right) t_{3} u_{3}^{+}-6 \tilde{F}\left(x, t_{3} v_{3}^{+}\right)\right) d x+\frac{1}{6} \int_{\Omega}\left(\tilde{f}\left(x, t_{4} u_{3}^{-}\right) t_{4} u_{3}^{-}-6 \tilde{F}\left(x, t_{4} u_{3}^{-}\right)\right) d x \\
& \leq \sum_{i=1}^{2}\left(\frac{1}{3}\left\|v_{i}\right\|^{2}+\frac{1}{6} \int_{\Omega}\left(\tilde{f}\left(x, v_{i}\right) v_{i}-6 \tilde{F}\left(x, v_{i}\right)\right) d x\right)+\frac{1}{3}\left\|u_{3}^{+}\right\|^{2}+\frac{1}{3}\left\|u_{3}^{-}\right\|^{2} \\
& +\frac{1}{6} \int_{\Omega}\left(\tilde{f}\left(x, u_{3}^{+}\right) u_{3}^{+}-6 \tilde{F}\left(x, u_{3}^{+}\right)\right) d x+\frac{1}{6} \int_{\Omega}\left(\tilde{f}\left(x, v_{3}^{-}\right) v_{3}^{-}-6 \tilde{F}\left(x, v_{3}^{-}\right)\right) d x .
\end{aligned}
$$

By using (2.14)-(2.16) and the fact that $u^{+}=w_{1}, u^{-}=w_{2}$, we have

$$
\begin{aligned}
m & \leq J\left(\bar{t}_{1} v_{1}+\bar{t}_{2} v_{2}+\bar{t}_{3} u^{+}+\bar{t}_{4} u^{-}\right) \\
& <\sum_{i=1}^{2}\left(\frac{1}{3}\left\|v_{i}\right\|^{2}+\frac{1}{6} \int_{\Omega}\left(\tilde{f}\left(x, v_{i}\right) v_{i}-6 \tilde{F}\left(x, v_{i}\right)\right) d x\right)+\frac{1}{3}\left\|u^{+}\right\|^{2}+\frac{1}{3}\left\|u^{-}\right\|^{2} \\
& +\frac{1}{6} \int_{\Omega}\left(\tilde{f}\left(x, u^{+}\right) u^{+}-6 \tilde{F}\left(x, u^{+}\right)\right) d x+\frac{1}{6} \int_{\Omega}\left(\tilde{f}\left(x, u^{-}\right) u^{-}-6 \tilde{F}\left(x, u^{-}\right)\right) d x \\
& +\frac{1}{2} \int_{\Omega}\left|\nabla w_{3}\right|^{2} d x+\frac{1}{2} \int_{\Omega}\left(\int_{0}^{|x|} \frac{s}{2}\left(v_{1}^{2}(s)+v_{2}^{2}(s)+w_{1}^{2}(s)+w_{2}^{2}(s)+w_{3}^{2}(s)\right) d s\right)^{2} \frac{w_{3}^{2}}{|x|^{2}} d x \\
& +\frac{1}{2} \int_{\Omega} \frac{v_{1}^{2}+v_{2}^{2}+w_{1}^{2}+w_{2}^{2}}{|x|^{2}}\left(\int _ { 0 } ^ { | x | } \frac { s } { 2 } \left(v_{1}^{2}(s)+v_{2}^{2}(s)+w_{1}^{2}(s)\right.\right. \\
& \left.\left.+w_{2}^{2}(s)\right) d s\right)\left(\int_{0}^{|x|} s w_{3}^{2}(s) d s\right) d x \\
& +\frac{1}{2} \int_{\Omega} \frac{v_{1}^{2}+v_{2}^{2}+w_{1}{ }^{2}+w_{2}{ }^{2}+w_{3}{ }^{2}}{|x|^{2}}\left(\int_{0}^{|x|} \frac{s}{2} w_{3}^{2}(s) d s\right)^{2} d x-\int_{\Omega} \tilde{F}\left(x, w_{3}\right) d x \\
& =J(v)=m,
\end{aligned}
$$

which is a contradiction. So $w_{3}=0$, and $v_{3}$ has indeed two nodal domains.

\section{Penalization of the nonlinearity and $L^{\infty}$-estimation}

In this section, we will modify the functional $I_{\lambda}$ by penalizing the nonlinearity $f(u)$. It plays a key role in establishing the relation between $m_{\lambda}$ and $m$ (will be defined later). On the other hand, we also give a delicate $L^{\infty}$-estimation for the critical points of the modified functional.

Since

$$
\Omega=\cup_{i=1}^{3} \Omega_{i} \text { and } \operatorname{dist}\left(\Omega_{i}, \Omega_{j}\right)>0 \text { for } i \neq j, i, j=1,2,3,
$$


there exist open sets $\Omega_{i}^{\rho}=\left\{x \in \mathbb{R}^{2}: \operatorname{dist}\left(x, \Omega_{i}\right)<\rho\right\}$ for $i=1,2,3$ with smooth boundary such that $\operatorname{dist}\left(\Omega_{i}^{\rho}, \Omega_{j}^{\rho}\right)>0$ for $i \neq j, i, j=1,2$, 3. Denote $\Omega^{\rho}:=\cup_{i=1}^{3} \Omega_{i}^{\rho}$. For open set $\Theta \subset \mathbb{R}^{2}$, we define

$$
H_{\lambda}(\Theta):=\left\{u \in H_{r}^{1}(\Theta) \mid \int_{\Theta} V(|x|) u^{2} d x<+\infty \text { and } u=0 \text { in } \mathbb{R}^{2} \backslash \Theta\right\}
$$

with norm

$$
\|u\|_{\lambda, \Theta}^{2}=\int_{\Theta}|\nabla u|^{2}+\lambda V(|x|) u^{2} d x .
$$

By $\left(V_{1}\right)$ and $\left(V_{2}\right)$, there exists a positive constant $v_{0}$ such that

$$
v_{0} \int_{\mathbb{R}^{2} \backslash \Omega^{\rho}} u^{2} d x \leq \frac{1}{2}\|u\|_{\lambda, \mathbb{R}^{2} \backslash \Omega^{\rho}}^{2} \text { for all } u \in H_{\lambda}\left(\mathbb{R}^{2} \backslash \Omega^{\rho}\right) .
$$

Let $a_{0}>0$ satisfy

$$
0<\max \left\{\frac{f\left(a_{0}\right)}{a_{0}},-\frac{f\left(-a_{0}\right)}{a_{0}}\right\} \leq v_{0},
$$

and $\tilde{f}, \tilde{F}: \mathbb{R} \rightarrow \mathbb{R}$ are the functions given by

$$
\tilde{f}(s)= \begin{cases}-\frac{f\left(-a_{0}\right)}{a_{0}} s, & \text { if } s<-a_{0}, \\ f(s), & \text { if }|s| \leq a_{0}, \\ \frac{f\left(a_{0}\right)}{a_{0}} s, & \text { if } s>a_{0},\end{cases}
$$

and

$$
\tilde{F}(s)=\int_{0}^{s} \tilde{f}(\tau) d \tau
$$

Using the above notations, we denote

$$
g(|x|, s):=\chi_{\Omega^{\rho}}(|x|) f(s)+\left(1-\chi_{\Omega^{\rho}}(|x|)\right) \tilde{f}(s)
$$

and

$$
G(|x|, s):=\int_{0}^{s} g(|x|, t) d t=\chi_{\Omega^{\rho}}(|x|) F(s)+\left(1-\chi_{\Omega^{\rho}}(|x|)\right) \tilde{F}(s),
$$

where $\chi_{\Omega^{\rho}}$ denotes the characteristic function of the set $\Omega^{\rho}$. We define the functional $\Phi_{\lambda}: H_{\lambda} \rightarrow \mathbb{R}$

$$
\Phi_{\lambda}(u)=\frac{1}{2} \int_{\mathbb{R}^{2}}|\nabla u|^{2}+\lambda V(|x|) u^{2} d x+\frac{1}{2} \int_{\mathbb{R}^{2}} \frac{u^{2}}{|x|^{2}}\left(\int_{0}^{|x|} \frac{s}{2} u^{2}(s) d s\right)^{2} d x-\int_{\mathbb{R}^{2}} G(|x|, u) d x
$$

and the critical points of $\Phi_{\lambda}$ are weak solutions of

$$
-\Delta u+\lambda V(|x|) u+\left(\frac{h^{2}(|x|)}{|x|^{2}}+\int_{|x|}^{+\infty} \frac{h(s)}{s} u^{2}(s) d s\right) u=g(|x|, u), \quad x \in \mathbb{R}^{2} .
$$

The next Proposition is about the asymptotic behavior of the critical points of $\Phi_{\lambda}$ as $\lambda \rightarrow+\infty$.

Proposition 3.1 Suppose $\lambda_{n} \rightarrow+\infty$ as $n \rightarrow \infty$ and $\left\{u_{\lambda_{n}}\right\} \subset H_{\lambda_{n}}$ satisfying

$$
\Phi_{\lambda_{n}}\left(u_{\lambda_{n}}\right) \rightarrow c \text { and }\left\|\Phi_{\lambda_{n}}^{\prime}\left(u_{\lambda_{n}}\right)\right\|_{H_{\lambda_{n}}^{\star}} \rightarrow 0 .
$$

Then, up to a subsequence, there exists $u \in H_{r}^{1}\left(\mathbb{R}^{2}\right)$ such that

(i) $\left\|u_{n}-u\right\|_{\lambda_{n}} \rightarrow 0$, consequently $u_{\lambda_{n}} \rightarrow u$ in $H_{r}^{1}\left(\mathbb{R}^{2}\right)$; 
(ii) $u=0$ in $\mathbb{R}^{2} \backslash \Omega$ and $u$ is a solution to equation (1.9);

(iii) $\Phi_{\lambda_{n}}\left(u_{\lambda_{n}}\right) \rightarrow J(u)=\frac{1}{2} \int_{\Omega}|\nabla u|^{2} d x+\frac{1}{2} \int_{\Omega} \frac{u^{2}}{|x|^{2}}\left(\int_{0}^{|x|} \frac{s}{2} u^{2}(s) d s\right)^{2} d x-\int_{\Omega} F(u) d x$.

Proof. It is easy to know that $\left\{u_{\lambda_{n}}\right\}$ is bounded in $H_{\lambda_{n}}\left(\mathbb{R}^{2}\right)$ and hence $\left\{u_{\lambda_{n}}\right\}$ is bounded in $H_{r}^{1}\left(\mathbb{R}^{2}\right)$. Passing to a subsequences, one has

$$
\begin{aligned}
& u_{n} \rightarrow u \text { weakly in } H_{r}^{1}\left(\mathbb{R}^{2}\right) ; \\
& u_{n} \rightarrow u \text { strongly in } L^{p}\left(\mathbb{R}^{N}\right) \text { for } 2<p<\infty ; \\
& u_{n} \rightarrow u \text { a.e. in } \mathbb{R}^{2} .
\end{aligned}
$$

We prove (ii) firstly. Let $m \in \mathbb{N}^{+}$, set $S_{m}=\left\{x \in \mathbb{R}^{2}: V(|x|) \geq \frac{1}{m}\right\}$, one has

$$
\int_{S_{m}} u_{\lambda_{n}}^{2} d x \leq \frac{2 m}{\lambda_{n}} \int_{S_{m}} \lambda_{n} V(|x|) u_{\lambda_{n}}^{2} d x \leq \frac{2 m C}{\lambda_{n}},
$$

and hence

$$
\int_{S_{m}} u^{2} d x \leq \liminf _{n \rightarrow \infty} \int_{S_{m}} u_{\lambda_{n}}^{2} d x=0 .
$$

It implies that $u \equiv 0$ in $S_{m}$. So we prove the $u \equiv 0$ in $\mathbb{R}^{2} \backslash \bar{\Omega}$. Now, for any $\varphi \in C_{0}^{\infty}(\Omega)$, since $\left\langle\Phi_{\lambda_{n}}^{\prime}\left(u_{n}\right), \varphi\right\rangle=0$, we can deduce that $u$ is a solution to equation (1.9).

Next, we prove $u_{\lambda_{n}} \rightarrow u$ in $H_{r}^{1}\left(\mathbb{R}^{2}\right)$. For convenience, let

$$
Y(u)=\frac{1}{2} \int_{\mathbb{R}^{2}} \frac{u^{2}}{|x|^{2}}\left(\int_{0}^{|x|} \frac{s}{2} u^{2}(s) d s\right)^{2} d x
$$

and $u_{n}=u_{\lambda_{n}}$. By virtue of $\left\langle\Phi_{\lambda_{n}}^{\prime}\left(u_{n}\right), u_{n}-u\right\rangle=\left\langle\Phi_{\lambda_{n}}^{\prime}(u), u_{n}-u\right\rangle=0$ when $n \rightarrow+\infty$, we have

$$
\begin{gathered}
\left(u_{n}, u_{n}-u\right)_{\lambda_{n}}+\left\langle Y^{\prime}\left(u_{n}\right), u_{n}-u\right\rangle=\int_{\mathbb{R}^{2}} g\left(|x|, u_{n}\right)\left(u_{n}-u\right) d x, \\
\left(u, u_{n}-u\right)_{\lambda_{n}}+\left\langle Y^{\prime}(u), u_{n}-u\right\rangle=\int_{\mathbb{R}^{2}} g(|x|, u)\left(u_{n}-u\right) d x .
\end{gathered}
$$

There, by Lemma 3.2 in [6], we have

$$
\lim _{n \rightarrow \infty}\left(\left\langle Y^{\prime}\left(u_{n}\right), u_{n}-u\right\rangle-\left\langle Y^{\prime}(u), u_{n}-u\right\rangle\right)=0 .
$$

Using the standard argument, we can deduce that

$$
\lim _{n \rightarrow \infty} \int_{\mathbb{R}^{2}} g\left(|x|, u_{n}\right)\left(u_{n}-u\right)-g(|x|, u)\left(u_{n}-u\right) d x=0 .
$$

Therefore, by (3.6), (3.7) and (3.8), we get

$$
\lim _{n \rightarrow \infty}\left\|u_{n}-u\right\|_{\lambda_{n}}^{2}=0
$$

On the other hand, the embedding $H_{\lambda} \hookrightarrow H_{r}^{1}\left(\mathbb{R}^{2}\right)$ is continuous. Indeed, by $\left(V_{1}\right)$ and $\left(V_{2}\right)$, we can deduce that

$$
V(x) \geq \delta_{0}>0, \quad x \in \mathbb{R}^{2} \backslash \Omega^{\rho},
$$

so it is easy to get

$$
\int_{\mathbb{R}^{2} \backslash \Omega^{\rho}}|\nabla u|^{2}+u^{2} d x \leq C \int_{\mathbb{R}^{2}}|\nabla u|^{2}+\lambda V(|x|) u^{2} d x .
$$


Thus we only need to show that

$$
\int_{\Omega^{\rho}}|\nabla u|^{2}+u^{2} d x \leq C \int_{\mathbb{R}^{2}}|\nabla u|^{2}+\lambda V(|x|) u^{2} d x .
$$

We choose a cut-off function $\Psi \in C^{\infty}\left(\mathbb{R}^{2}\right)$ such that $0 \leq \Psi \leq 1$ in $\mathbb{R}^{2}, \Psi(x)=1$ for each $x \in \Omega^{\rho}$ and $\Psi(x)=0$ for $x \in \mathbb{R}^{2} \backslash \Omega^{2 \rho}$ and $|\nabla \Psi|<C$. By Sobolev's embedding inequality,

$$
\begin{aligned}
\int_{\Omega^{\rho}} u^{2} d x & \leq \int_{\Omega^{2 \rho}}|u \Psi|^{2} d x \leq C \int_{\Omega^{2 \rho}}|\nabla(u \Psi)|^{2} d x \\
& \leq 2 C \int_{\Omega^{2 \rho}}|\nabla u|^{2} d x+2 C \int_{\Omega^{2 \rho} \backslash \Omega^{\rho}} u^{2} d x \\
& \leq C \int_{\mathbb{R}^{2}}|\nabla u|^{2}+\lambda V(|x|) u^{2} d x .
\end{aligned}
$$

So we get $\int_{\mathbb{R}^{2}}|\nabla u|^{2}+u^{2} d x \leq C \int_{\mathbb{R}^{2}}|\nabla u|^{2}+\lambda V(|x|) u^{2} d x$. Thus we can get that

$$
\left\|u_{n}-u\right\|_{H_{r}^{1}\left(\mathbb{R}^{2}\right)} \rightarrow 0 \text { as } n \rightarrow \infty .
$$

Combining with (i), it is easy to prove the (iii).

The next Lemma is important which indicates that the critical points $u_{\lambda}$ of $\Phi_{\lambda}$ with bounded energy are the solutions of the original problem (1.9) if $\lambda$ large enough.

Lemma 3.2. Fix $M>0$, for any critical points $u_{\lambda}$ of $\Phi_{\lambda}\left(u_{\lambda}\right) \leq M$. Then there exists $\Lambda_{0}>0$ such that $\lambda \geq \Lambda_{0}$, one has

$$
\left|u_{\lambda}(x)\right| \leq a_{0} \text { for all } x \in \mathbb{R}^{2} \backslash \Omega^{\rho} .
$$

Proof. We prove Lemma 3.2 by Moser's iteration. By Proposition 3.1, it is easy to get that

$$
\lim _{\lambda \rightarrow \infty} \int_{\mathbb{R}^{2} \backslash \Omega}\left|u_{\lambda}\right|^{q} d x=0, \forall 2<q<\infty .
$$

So, for any small $\eta_{0}>0$ and $\lambda$ large enough,

$$
\int_{\mathbb{R}^{2} \backslash \Omega}\left|u_{\lambda}\right|^{6} d x \leq 2 \eta_{0} .
$$

Let $\psi$ be a smooth cut-off function and $\beta>1$, both of them will be specified later. For $R>0$, we define

$$
u_{\lambda}^{R}= \begin{cases}R, & \text { if } u_{\lambda}>R, \\ u_{\lambda}, & \text { if }\left|u_{\lambda}\right| \leq R, \\ -R, & \text { if } u_{\lambda}<R\end{cases}
$$

and multiply (3.3) by $\psi^{2}\left|u_{\lambda}^{R}\right|^{\beta-1} u_{\lambda}$, then

$$
\begin{aligned}
& \int_{\mathbb{R}^{2}} \nabla\left(\psi^{2}\left|u_{\lambda}^{R}\right|^{\beta-1} u_{\lambda}\right) \nabla u_{\lambda} d x+\lambda \int_{\mathbb{R}^{2}} V(|x|) \psi^{2}\left|u_{\lambda}^{R}\right|^{\beta-1} u_{\lambda}^{2} d x \\
& +\int_{\mathbb{R}^{2}}\left(\frac{h^{2}(|x|)}{|x|^{2}}+\int_{|x|}^{+\infty} \frac{h(s)}{s} u^{2}(s) d s\right) \psi^{2}\left|u_{\lambda}^{R}\right|^{\beta-1} u_{\lambda}^{2} d x \\
& =\int_{\mathbb{R}^{2}} g\left(|x|, u_{\lambda}\right) \psi^{2}\left|u_{\lambda}^{R}\right|^{\beta-1} u_{\lambda} d x .
\end{aligned}
$$


That is

$$
\begin{aligned}
& \int_{\mathbb{R}^{2}} \psi^{2}\left|u_{\lambda}^{R}\right|^{\beta-1}\left|\nabla u_{\lambda}\right|^{2} d x+(\beta-1) \int_{\mathbb{R}^{2}} \psi^{2}\left|u_{\lambda}^{R}\right|^{\beta-3} u_{\lambda}^{R} u_{\lambda} \nabla u_{\lambda}^{R} \nabla u_{\lambda} d x \\
& \quad+2 \int_{\mathbb{R}^{2}} \psi\left|u_{\lambda}^{R}\right|^{\beta-1} u_{\lambda} \nabla \psi \nabla u_{\lambda} d x+\lambda \int_{\mathbb{R}^{2}} V(|x|) \psi^{2}\left|u_{\lambda}^{R}\right|^{\beta-1} u_{\lambda}^{2} d x \\
& \leq \int_{\mathbb{R}^{2}} g\left(|x|, u_{\lambda}\right) \psi^{2}\left|u_{\lambda}^{R}\right|^{\beta-1} u_{\lambda} d x .
\end{aligned}
$$

On the other hand, by Hölder's inequality and Young's inequality, one has

$$
\left.\left.\left|\int_{\mathbb{R}^{2}} \psi\right| u_{\lambda}^{R}\right|^{\beta-1} u_{\lambda} \nabla \psi \nabla u_{\lambda} d x\left|\leq \frac{1}{4} \int_{\mathbb{R}^{2}} \psi^{2}\right| u_{\lambda}^{R}\right|^{\beta-1}\left|\nabla u_{\lambda}\right|^{2} d x+C \int_{\mathbb{R}^{2}}|\nabla \psi|^{2}\left|u_{\lambda}^{R}\right|^{\beta-1} u_{\lambda}^{2} d x .
$$

Note $|g(|x|, u) u| \leq|u|^{2}+C_{0}|u|^{q_{0}}$, so the inequality (3.13) leads to

$$
\begin{aligned}
& \frac{1}{2} \int_{\mathbb{R}^{2}} \psi^{2}\left|u_{\lambda}^{R}\right|^{\beta-1}\left|\nabla u_{\lambda}\right|^{2} d x+(\beta-1) \int_{\mathbb{R}^{2}} \psi^{2}\left|u_{\lambda}^{R}\right|^{\beta-3} u_{\lambda}^{R} u_{\lambda} \nabla u_{\lambda}^{R} \nabla u_{\lambda} d x \\
& \leq 2 C \int_{\mathbb{R}^{2}}|\nabla \psi|^{2}\left|u_{\lambda}^{R}\right|^{\beta-1} u_{\lambda}^{2} d x+\int_{\mathbb{R}^{2}} \psi^{2} u_{\lambda}^{2}\left|u_{\lambda}^{R}\right|^{\beta-1} d x+C_{0} \int_{\mathbb{R}^{2}} \psi^{2} u_{\lambda}^{q_{0}}\left|u_{\lambda}^{R}\right|^{\beta-1} d x .
\end{aligned}
$$

By sobolev imbedding theorem, we have

$$
\begin{aligned}
& S(p)\left(\int_{\mathbb{R}^{2}}\left(\psi\left|u_{\lambda}^{R}\right|^{\frac{\beta-1}{2}} u_{\lambda}\right)^{p} d x\right)^{\frac{2}{p}} \leq \int_{\mathbb{R}^{2}}\left|\nabla\left(\psi\left|u_{\lambda}^{R}\right|^{\frac{\beta-1}{2}} u_{\lambda}\right)\right|^{2} d x \\
& \leq \frac{(\beta+1)^{2}}{2} \int_{\mathbb{R}^{2}} \psi^{2}\left|u_{\lambda}^{R}\right|^{\beta-1}\left|\nabla u_{\lambda}\right|^{2} d x+2 \int_{\mathbb{R}^{2}}|\nabla \psi|^{2}\left|u_{\lambda}^{R}\right|^{\beta-1} u_{\lambda}^{2} d x,
\end{aligned}
$$

where $S(p)$ is imbedding constant. Using (3.14) and (3.15), one has

$$
\begin{aligned}
& S(p)\left(\int_{\mathbb{R}^{2}}\left(\psi\left|u_{\lambda}^{R}\right|^{\frac{\beta-1}{2}} u_{\lambda}\right)^{p} d x\right)^{\frac{2}{p}} \leq\left(2+2(\beta+1)^{2} C\right) \int_{\mathbb{R}^{2}}|\nabla \psi|^{2}\left|u_{\lambda}^{R}\right|^{\beta-1} u_{\lambda}^{2} d x \\
& +C_{0}(\beta+1)^{2} \int_{\mathbb{R}^{2}} \psi^{2} u_{\lambda}^{q_{0}}\left|u_{\lambda}^{R}\right|^{\beta-1} d x+(\beta+1)^{2} \int_{\mathbb{R}^{2}} \psi^{2}\left|u_{\lambda}^{R}\right|^{\beta-1} u_{\lambda}^{2} d x .
\end{aligned}
$$

Now, for $y \in \mathbb{R}^{2} \backslash \Omega^{\rho}$ and fix a $r$ which $0<r<\frac{\rho}{8}$. Then take the cut-off function $\psi$ by

$$
\psi(x)= \begin{cases}1, & x \in B_{2 r}(y) \\ 0, & \mathbb{R}^{2} \backslash B_{4 r}(y)\end{cases}
$$

and $0 \leq \psi \leq 1,|\nabla \psi| \leq \frac{C}{r}$. Using Hölder's inequality and (3.10), we have

$$
\begin{aligned}
\int_{\mathbb{R}^{2}} \psi^{2} u_{\lambda}^{q_{0}}\left|u_{\lambda}^{R}\right|^{\beta-1} d x & \leq\left(\int_{\mathbb{R}^{2}}\left(\psi^{2}\left|u_{\lambda}^{R}\right|^{\beta-1} u_{\lambda}^{2}\right)^{\frac{p}{2}} d x\right)^{\frac{2}{p}}\left(\int_{\mathbb{R}^{2} \backslash \Omega}\left(u_{\lambda}^{q_{0}-2}\right)^{\frac{p}{p-2}} d x\right)^{\frac{p-2}{p}} \\
& \leq \frac{S(p)}{2 C_{0}(\beta+1)^{2}}\left(\int_{\mathbb{R}^{2}}\left(\psi^{2}\left|u_{\lambda}^{R}\right|^{\beta-1} u_{\lambda}^{2}\right)^{\frac{p}{2}} d x\right)^{\frac{2}{p}} .
\end{aligned}
$$


Combing (3.16) and (3.17), we have

$$
\begin{aligned}
& S(p)\left(\int_{\mathbb{R}^{2}}\left(\psi\left|u_{\lambda}^{R}\right|^{\frac{\beta-1}{2}} u_{\lambda}\right)^{p} d x\right)^{\frac{2}{p}} \leq\left(4+4(\beta+1)^{2} C\right) \int_{\mathbb{R}^{2}}|\nabla \psi|^{2}\left|u_{\lambda}^{R}\right|^{\beta-1} u_{\lambda}^{2} d x \\
& \quad+2(\beta+1)^{2} \int_{\mathbb{R}^{2}} \psi^{2}\left|u_{\lambda}^{R}\right|^{\beta-1} u_{\lambda}^{2} d x .
\end{aligned}
$$

Taking limit $R \rightarrow+\infty$ and $\beta=5$ in (3.18), which implies that for any $y \in \mathbb{R}^{2} \backslash \Omega^{\rho}$,

$$
\left(\int_{B_{2 r}(y)}\left|u_{\lambda}\right|^{3 p} d x\right)^{\frac{2}{p}} \leq C(r, p) \int_{B_{4 r}(y)}\left|u_{\lambda}\right|^{6} d x
$$

Because $p \in(2,+\infty)$, we choose $p=\frac{3}{2} q_{0}-3>2$ since $q_{0}>4$, one has

$$
\left(\int_{B_{2 r}(y)}\left|u_{\lambda}\right|^{\frac{9}{2} q_{0}-9} d x\right)^{\frac{4}{3 q_{0}-6}} \leq C(r) \int_{B_{4 r}(y)}\left|u_{\lambda}\right|^{6} d x
$$

Now, we use the above estimation combining with Moser's iteration argument to complete the proof. Let $Z_{\lambda}=\left|u_{\lambda}^{R}\right|^{\frac{\beta-1}{2}} u_{\lambda}$, where $\beta>1$ will choose later, then (3.16) becomes

$$
\begin{aligned}
& S(6)\left(\int_{\mathbb{R}^{2}}\left(\psi Z_{\lambda}\right)^{6} d x\right)^{\frac{1}{3}} \leq\left(2+2(\beta+1)^{2} C\right) \int_{\mathbb{R}^{2}}|\nabla \psi|^{2} Z_{\lambda}^{2} d x \\
& +C_{0}(\beta+1)^{2} \int_{\mathbb{R}^{2}} \psi^{2} Z_{\lambda}^{2} u_{\lambda}^{q_{0}-2} d x+(\beta+1)^{2} \int_{\mathbb{R}^{2}} \psi^{2} Z_{\lambda}^{2} d x,
\end{aligned}
$$

where $\psi$ is a cut-off function supported in $B_{2 r}(y)$ with $y \in \mathbb{R}^{3} \backslash \Omega^{\rho}$ and $r \leq \frac{\rho}{4}$. By the Hölder's inequality, we get

$$
\int_{\mathbb{R}^{2}} \psi^{2} Z_{\lambda}^{2} u_{\lambda}^{q_{0}-2} d x \leq\left(\int_{\mathbb{R}^{2}}\left(\psi Z_{\lambda}\right)^{\frac{18}{7}} d x\right)^{\frac{7}{9}}\left(\int_{B_{2 r}(y)} u_{\lambda}^{\frac{9}{2} q_{0}-9} d x\right)^{\frac{2}{9}} .
$$

Since $2<\frac{18}{7}<6$, thus, for any $\epsilon>0$,

$$
\left\|\psi Z_{\lambda}\right\|_{\frac{18}{7}}^{2} \leq \epsilon\left\|\psi Z_{\lambda}\right\|_{6}^{2}+\epsilon^{-\frac{1}{2}}\left\|\psi Z_{\lambda}\right\|_{2}^{2} .
$$

By (3.21) and above estimate, it deduces that

$$
\begin{aligned}
& S(6)\left(\int_{\mathbb{R}^{2}}\left(\psi Z_{\lambda}\right)^{6} d x\right)^{\frac{1}{3}} \leq\left(2+2(\beta+1)^{2} C\right) \int_{\mathbb{R}^{2}}|\nabla \psi|^{2} Z_{\lambda}^{2} d x \\
& +C_{0} C_{1}(r)(\beta+1)^{2}\left(\epsilon\left\|\psi Z_{\lambda}\right\|_{6}^{2}+\epsilon^{-\frac{1}{2}}\left\|\psi Z_{\lambda}\right\|_{2}^{2}\right)+(\beta+1)^{2} \int_{\mathbb{R}^{2}} \psi^{2} Z_{\lambda}^{2} d x,
\end{aligned}
$$

where

$$
C_{1}(r)=\left(\int_{B_{2 r}(y)} u_{\lambda}^{\frac{9}{2} q_{0}-9} d x\right)^{\frac{2}{9}} \leq\left(C(r) \int_{B_{4 r}(y)}\left|u_{\lambda}\right|^{6} d x\right)^{\frac{q_{0}-2}{6}} \leq\left[2 \eta_{0} C(r)\right]^{\frac{q_{0}-2}{6}} .
$$

Setting $\epsilon=S(6)\left[2 C_{0} C_{1}(r)(\beta+1)^{2}\right]^{-1}$, we obtain from (3.22) that

$$
\left(\int_{\mathbb{R}^{2}}\left(\psi Z_{\lambda}\right)^{6} d x\right)^{\frac{1}{3}} \leq \frac{\left(4+4(\beta+1)^{2} C\right)}{S} \int_{\mathbb{R}^{2}}|\nabla \psi|^{2} Z_{\lambda}^{2} d x+\frac{C_{2}}{r^{\frac{q_{0}-2}{4}}}(\beta+1)^{3} \int_{\mathbb{R}^{2}} \psi^{2} Z_{\lambda}^{2} d x .
$$


Now, for $r \leq r_{2}<r_{1} \leq 2 r$, we choose $\psi$ such that $\psi \equiv 1$ in $B_{r_{2}}(y), \psi \equiv 0$ in $\mathbb{R}^{2} \backslash B_{r_{1}}(y)$ and $0 \leq \psi \leq 1$, $|\nabla \psi| \leq \frac{C}{r_{1}-r_{2}}$. Then we obtain that

$$
\left\|Z_{\lambda}\right\|_{L^{6}\left(B_{r_{2}(y)}\right)} \leq \frac{C_{3}}{\left(r_{1}-r_{2}\right)} h^{\frac{3}{2}}\left(r^{\frac{q_{0}-2}{4}}\right)^{-\frac{1}{2}}\left\|Z_{\lambda}\right\|_{L^{2}\left(B_{r_{1}(y)}\right)},
$$

where $h=(1+\beta)$. Set

$$
L(p, r):=\left(\int_{B_{r}(y)}\left|u_{\lambda}\right|^{p} d x\right)^{\frac{1}{p}} .
$$

When $R \rightarrow+\infty$ in (3.24), then we have

$$
L\left(3 h, r_{2}\right) \leq\left(\frac{C_{3}}{\left(r_{1}-r_{2}\right)}\right)^{\frac{2}{h}} h^{\frac{3}{h}}\left(r^{\frac{q_{0}-2}{4}}\right)^{-\frac{1}{h}} L\left(h, r_{1}\right) .
$$

Let $h=h_{m}=6 \cdot 3^{m}, r_{m}=r\left(1+2^{-m}\right)$ for $m=0,1,2, \cdots$, by (3.25), we get

$$
\begin{aligned}
L\left(6 \cdot 3^{m+1}, r^{m+1}\right) & =L\left(3 h_{m}, r_{m+1}\right) \leq\left(\frac{C_{3}}{r_{m}-r_{m+1}}\right)^{\frac{2}{h_{m}}} h_{m}^{\frac{3}{h_{m}}}\left(r^{\frac{q_{0}-2}{4}}\right)^{-\frac{1}{h_{m}}} L\left(h_{m}, r_{m}\right) \\
& =\left(\frac{2 C_{3}}{r^{q_{0}+6}} 6^{\frac{3}{2}}\right)^{\frac{1}{3 \cdot 3 m}}\left(2 \cdot 3^{\frac{3}{2}}\right)^{\frac{m}{3 \cdot 3^{m}}} L\left(h_{m}, r_{m}\right) \leq \cdots \\
& \leq\left(\frac{2 C_{3}}{r^{\frac{q_{0}+6}{8}}} 6^{\frac{3}{2}}\right)^{\frac{1}{3} \sum_{j=0}^{\infty} 3^{-j}}\left(2 \cdot 3^{\frac{3}{2}}\right)^{\frac{1}{3} \sum_{j=0}^{\infty} j 3^{-j}} L(6,2 r) .
\end{aligned}
$$

Let $m \rightarrow \infty$, we have

$$
\sup _{x \in B_{r}(y)}|u(x)|=\lim _{s \rightarrow \infty} L(s, r) \leq C_{4}(r)\left(2 \eta_{0}\right)^{\frac{1}{6}} .
$$

We can choose $\Lambda_{0}$, when $\lambda \geq \Lambda_{0}$, we have $C_{4}(r)\left(2 \eta_{0}\right)^{\frac{1}{6}} \leq a_{0}$. So we can get

$$
\left\|u_{\lambda_{n}}\right\|_{L^{\infty}\left(\mathbb{R}^{2} \backslash \Omega^{\rho}\right)} \leq a_{0}
$$

for $\lambda \geq \Lambda_{0}$. Therefore we complete the proof.

\section{A special minimax value for the modified functional}

We investigate a special minimax value for the modified functional $\Phi_{\lambda}$, which is used to get a key Lemma. We define a new functional

$$
\bar{J}_{\lambda}(u)=\frac{1}{2} \int_{\Omega^{\rho}}|\nabla u|^{2}+\lambda V(|x|) u^{2} d x+\frac{1}{2} \int_{\Omega^{\rho}} \frac{u^{2}}{|x|^{2}}\left(\int_{0}^{|x|} \frac{s}{2} u^{2}(s) d s\right)^{2} d x-\int_{\Omega^{\rho}} F(u) d x
$$

which is well defined and belongs to $C^{1}\left(H_{\lambda}\left(\Omega^{\rho}\right), \mathbb{R}\right)$. We define set

$$
\overline{\mathcal{M}}=\left\{u \in H_{\lambda}\left(\Omega^{\rho}\right) \mid\left\langle\bar{J}^{\prime}(u), u_{i}\right\rangle=0 \text { for } i=1,2, u_{1}^{+} \neq 0, u_{2}^{-} \neq 0 \text { and }\left\langle\bar{J}^{\prime}(u), u_{3}^{ \pm}\right\rangle=0, u_{3}^{ \pm} \neq 0\right\}
$$

and

$$
\bar{m}_{\lambda}:=\inf _{u \in \overline{\mathcal{M}}} \bar{J}_{\lambda}(u) .
$$

Similar to the proof of Section 2, we deduce that there exists $\bar{v}_{\lambda} \in H_{\lambda}\left(\Omega^{\rho}\right)$ such that

$$
\bar{J}_{\lambda}\left(\bar{v}_{\lambda}\right)=\bar{m}_{\lambda} \text { and } \bar{J}_{\lambda}^{\prime}\left(\bar{v}_{\lambda}\right)=0 \text {. }
$$

Lemma 4.1. There holds that

(i) $0<\bar{m}_{\lambda} \leq m$, for all $\lambda>0$; 
(ii) $\bar{m}_{\lambda} \rightarrow m$, as $\lambda \rightarrow+\infty$.

Proof. The proof of $(i)$ is trivial since $u \in \mathcal{M}$ which also belongs to $\overline{\mathcal{M}}$ by zero extension.

Now we are going to prove (ii). Let $\left\{\lambda_{n}\right\}$ be a sequence with $\lambda_{n} \rightarrow+\infty$. For each $\lambda_{n}$, there exists $u_{\lambda_{n}} \in$ $H_{\lambda_{n}}\left(\Omega^{\rho}\right)$ with

$$
\bar{J}_{\lambda_{n}}\left(u_{\lambda_{n}}\right)=\bar{m}_{\lambda_{n}} \text { and } \bar{J}_{\lambda_{n}}^{\prime}\left(u_{\lambda_{n}}\right)=0 .
$$

Since $\bar{m}_{\lambda_{n}} \leq m$, we can suppose $\left\{\bar{J}\left(u_{\lambda_{n}}\right)\right\}$ convergence (up to a subsequence) and $\bar{J}_{\lambda_{n}}^{\prime}\left(u_{\lambda_{n}}\right)=0$. It is easy to know that there exists $u \in H_{0}^{1}(\Omega) \cap H_{r}^{1}(\Omega) \subset H_{\lambda}\left(\Omega^{\rho}\right)$ such that

$$
u_{\lambda_{n}} \rightarrow u \text { in } H_{\lambda}\left(\Omega^{\rho}\right) \text { as } n \rightarrow+\infty
$$

and $\left(\left.u\right|_{\Omega_{1}}\right)^{+},\left(\left.u\right|_{\Omega_{2}}\right)^{-},\left(\left.u\right|_{\Omega_{3}}\right)^{ \pm} \neq 0$. Moreover,

$$
\bar{m}_{\lambda_{n}}=\bar{J}_{\lambda_{n}}\left(u_{\lambda_{n}}\right) \rightarrow J(u),
$$

and

$$
0=\bar{J}_{\lambda_{n}}^{\prime}\left(u_{\lambda_{n}}\right) \rightarrow J^{\prime}(u)
$$

By the definition of $m$, one has that

$$
\lim _{\lambda_{n} \rightarrow+\infty} \bar{m}_{\lambda_{n}}=J(u) \geq m .
$$

Using conclusion (i), we obtain that $\bar{m}_{\lambda_{n}} \rightarrow m$ as $n \rightarrow+\infty$.

In Section 2, we have known that there exists $v \in H$, that is

$$
v \in \mathcal{M}, J(v)=m, J^{\prime}(v)=0 .
$$

and $v_{1}=\left.v\right|_{\Omega_{1}}$ is positive, $v_{2}=\left.v\right|_{\Omega_{2}}$ is negative and $v_{3}=\left.v\right|_{\Omega_{3}}$ changes sign exactly once. At the same time, we can find two positive constants $\tau_{2}>\tau_{1}$ such that

$$
\tau_{1} \leq\left\|v_{1}\right\|,\left\|v_{2}\right\|,\left\|v_{3}^{+}\right\|,\left\|v_{3}^{-}\right\| \leq \tau_{2} .
$$

We define $y_{0}:\left[\frac{1}{2}, \frac{3}{2}\right] \rightarrow H_{\lambda}$ by

$$
y_{0}\left(t_{1}, t_{2}, t_{3}, t_{4}\right):=t_{1} v_{1}+t_{2} v_{2}+t_{3} v_{3}^{+}+t_{4} v_{3}^{-}
$$

and

$$
m_{\lambda}:=\inf _{y \in \Sigma_{\lambda}} \max _{t \in\left[\frac{1}{2}, \frac{3}{2}\right]^{4}} \Phi_{\lambda}(y(t))
$$

where

$$
\begin{gathered}
\Sigma_{\lambda}:=\left\{y \in C\left(\left[\frac{1}{2}, \frac{3}{2}\right]^{4}, H_{\lambda}\right):\|y(t)\|_{\lambda} \leq 6 \tau_{2}+\tau_{1},\left(\left.y\right|_{\Omega_{1}^{\rho}}\right)^{+},\left(\left.y\right|_{\Omega_{2}^{\rho}}\right)^{-},\left(\left.y\right|_{\Omega_{3}^{\rho}}\right)^{ \pm} \neq 0\right. \\
\text { and } \left.y=y_{0} \text { on } \partial\left[\frac{1}{2}, \frac{3}{2}\right]^{4}\right\} .
\end{gathered}
$$

It is easy to check $y_{0} \in \Sigma_{\lambda}$, so $\Sigma_{\lambda} \neq \emptyset$ and $m_{\lambda}$ is well defined.

The next Lemma is trivial by degree theory, so we omit the detail.

Lemma 4.2. For any $y \in \Sigma_{\lambda}$, there exists an 4-tuple $t^{\star}=\left(t_{1}^{\star}, t_{2}^{\star}, t_{3}^{\star}, t_{4}^{\star}\right) \in D=\left(\frac{1}{2}, \frac{3}{2}\right)^{4}$ such that $\left\langle\bar{J}_{\lambda}^{\prime}\left(\left.y\left(t^{\star}\right)\right|_{\Omega^{\rho}}\right), y_{1}^{+}\left(t^{\star}\right)\right\rangle=\left\langle\bar{J}_{\lambda}^{\prime}\left(\left.y\left(t^{\star}\right)\right|_{\Omega^{\rho}}\right), y_{2}^{-}\left(t^{\star}\right)\right\rangle=0$ and $\left\langle\bar{J}_{\lambda}^{\prime}\left(\left.y\left(t^{\star}\right)\right|_{\Omega^{\rho}}\right), y_{3}^{ \pm}\left(t^{\star}\right)\right\rangle=0$ where $y_{i}(t)=\left.y(t)\right|_{\Omega_{i}^{\rho}}$ for $i=1,2,3$.

Lemma 4.3. There holds that

(i) $\bar{m}_{\lambda} \leq m_{\lambda} \leq m$ for all $\lambda \geq 1$;

(ii) $m_{\lambda} \rightarrow m$ as $\lambda \rightarrow+\infty$;

(iii) There exists $\epsilon_{0}>0$ such that $\Phi_{\lambda}(y(t))<m-\epsilon_{0}$ for all $\lambda \geq 0, y \in \Sigma_{\lambda}$ and $t=\left(t_{1}, t_{2}, t_{3}, t_{4}\right) \in \partial\left[\frac{1}{2}, \frac{3}{2}\right]^{4}$. 
Proof. (i) Since $y_{0} \in \Sigma_{\lambda}$, we have

$$
m_{\lambda} \leq \max _{t \in\left[\frac{1}{2}, \frac{3}{2}\right]^{4}} \Phi_{\lambda}\left(y_{0}(t)\right)=\max _{t \in\left[\frac{1}{2}, \frac{3}{2}\right]^{4}} J\left(y_{0}(t)\right)=m .
$$

Now, fixing $t^{\star} \in\left(\frac{1}{2}, \frac{3}{2}\right)^{4}$ given by Lemma 4.2, it implies

$$
\bar{m}_{\lambda} \leq \bar{J}_{\lambda}\left(\left.y\left(t^{\star}\right)\right|_{\Omega^{\rho}}\right) .
$$

By the definition of $g(|x|, u)$, we deduce that $|G(|x|, u)| \leq \frac{v_{0}}{2} u^{2}$ for $x \in \mathbb{R}^{2} \backslash \Omega^{\rho}$. By (3.1) we can get

$$
\Phi_{\lambda}\left(y\left(t^{\star}\right)\right) \geq \bar{J}_{\lambda}\left(\left.y\left(t^{\star}\right)\right|_{\Omega^{\rho}}\right) .
$$

Therefore,

$$
\max _{t \in\left[\frac{1}{2}, \frac{3}{2}\right]^{4}} \Phi_{\lambda}(y(t)) \geq \bar{J}_{\lambda}\left(\left.y\left(t^{*}\right)\right|_{\Omega^{\rho}}\right) \geq \bar{m}_{\lambda} \text {, for each } y \in \Sigma_{\lambda} .
$$

So $m_{\lambda} \geq \bar{m}_{\lambda}$.

(ii) it is obtained by Lemma 4.1 (ii) and Lemma 4.3 (i).

(iii) For $t=\left(t_{1}, t_{2}, t_{3}, t_{4}\right) \in \partial\left[\frac{1}{2}, \frac{3}{2}\right]^{4}$, we have

$$
\Phi_{\lambda}(y(t))=J\left(y_{0}(t)\right) \text { for } t=\left(t_{1}, t_{2}, t_{3}, t_{4}\right) \in \partial\left[\frac{1}{2}, \frac{3}{2}\right]^{4} .
$$

By Lemma 2.1, it is to get

$$
\Phi_{\lambda}(y(t))<m-\epsilon_{0} \text { for } t=\left(t_{1}, t_{2}, t_{3}, t_{4}\right) \in \partial\left[\frac{1}{2}, \frac{3}{2}\right]^{4},
$$

where $\epsilon_{0}$ is a small positive constant.

\section{Proof of Theorem 1.1}

In this section, we prove our main results. Define

$$
S:=\{u \in \mathcal{M} \mid J(u)=m\} .
$$

Then we need further to study the properties of the set $S$.

Lemma 5.1. $S$ is compact in $H$.

Proof. The proof is standard, we omit it immediately.

Lemma 5.2. Let $d>0$ be a fixed number and let $\left\{u_{n}\right\} \subset S^{d}$ be a sequence. Then, up to a subsequence, $u_{n} \rightarrow u_{0}$ in $H_{\lambda}$ as $n \rightarrow \infty$, and $u_{0} \in S^{2 d}$ where

$$
S^{d}:=\left\{u \in H_{\lambda}: \operatorname{dist}_{\lambda}(u, S) \leq d\right\}
$$

and dist ${ }_{\lambda}$ denotes the distance in $H_{\lambda}$.

Proof. Since $S$ is compact in $H$, we can choose $\left\{\bar{u}_{n}\right\} \subset S$ satisfy

$$
\operatorname{dist}_{\lambda}\left(u_{n}, \bar{u}_{n}\right) \leq d \text {. }
$$

On the other hand, there exists $\bar{u} \in S$ such that, up to a subsequence, $\bar{u}_{n} \rightarrow \bar{u}$ in $H$. Hence, $\operatorname{dist}\left(u_{n}, u\right) \leq d$ for $n$ large enough. Thus $\left\{u_{n}\right\}$ is bounded in $H_{\lambda}$. Up to a subsequence, $u_{n} \rightarrow u_{0}$ weakly in $H_{\lambda}$. Since $B_{2 d}(u)$ is weakly closed in $H_{\lambda}$, so $u_{0} \in B_{2 d}(u) \subset S^{2 d}$.

Lemma 5.3. Let $d \in\left(0, \tau_{1}\right)$, where $\tau_{1}$ is given by (4.4). Suppose that there exist a sequence $\lambda_{n}>0$ with $\lambda_{n} \rightarrow \infty$, and $\left\{u_{n}\right\} \subset S^{d}$ satisfying

$$
\lim _{n \rightarrow \infty} \Phi_{\lambda_{n}}\left(u_{n}\right) \leq m, \lim _{n \rightarrow \infty} \Phi_{\lambda_{n}}^{\prime}\left(u_{n}\right)=0 .
$$

Then, up to a subsequence, $\left\{u_{n}\right\}$ converges strongly in $H_{r}^{1}\left(\mathbb{R}^{2}\right)$ to an element $u \in S$. 
Proof. Observe that, by $\lim _{n \rightarrow \infty} \Phi_{\lambda_{n}}\left(u_{n}\right) \leq m$ and $\lim _{n \rightarrow \infty} \Phi_{\lambda_{n}}^{\prime}\left(u_{n}\right)=0$ we deduce that $\left\{\left\|u_{u}\right\|_{\lambda_{n}}\right\}$ and $\left\{\Phi_{\lambda_{n}}\left(u_{n}\right)\right\}$ are bounded. Up to a subsequence, we may assume that $\Phi_{\lambda_{n}}\left(u_{n}\right) \rightarrow c \leq m$. By Proposition 3.1, there exists $u \in H_{r}^{1}\left(\mathbb{R}^{2}\right)$ such that $u_{n} \rightarrow u$ in $H_{r}^{1}\left(\mathbb{R}^{2}\right), u=0$ in $\mathbb{R}^{2} \backslash \Omega$ and $\Phi_{\lambda_{n}}\left(u_{n}\right) \rightarrow J(u)$. Moreover, $u$ is a solution of equation (1.9). Next we prove that $u \in S$. Since $\left\{u_{n}\right\} \subset S^{d}$ and $d \in\left(0, \tau_{1}\right)$, we can deduce that $\left(\left.u\right|_{\Omega^{1}}\right)^{+} \neq 0$, $\left(\left.u\right|_{\Omega^{2}}\right)^{-} \neq 0$ and $\left(\left.u\right|_{\Omega^{3}}\right)^{ \pm} \neq 0$. Indeed, if the conclusion is not correct, we can assume $\left(\left.u\right|_{\Omega^{1}}\right)^{+}=0$, we can choose $\left\{\bar{u}_{n}\right\} \subset S$ satisfy

$$
\operatorname{dist}_{\lambda}\left(u_{n}, \bar{u}_{n}\right) \leq d,
$$

so

$$
\tau_{1} \leq\left\|\bar{u}_{n, 1}\right\| \leq\left\|\bar{u}_{n, 1}-u_{n, 1}\right\|_{H_{\lambda_{n}}}+\left\|u_{n, 1}\right\| \leq d,
$$

which implies that a contradiction. Hence, by Proposition 3.1 again, we get $J^{\prime}(u)=0, u=0$ in $\mathbb{R}^{2} \backslash \Omega$. Then we get that $J(u) \geq m$. At the same time, $\Phi_{\lambda_{n}}\left(u_{n}\right) \rightarrow J(u) \leq m$, therefore $u \in S$.

Lemma 5.4. Let $\delta \in\left(0, \tau_{1}\right)$, where $\tau_{1}$ is given by (4.4). Then there exist constants $0<\sigma<1$ and $\Lambda_{1}>0$ such that $\left\|\Phi_{\lambda}^{\prime}(u)\right\|_{H_{\lambda}^{*}} \geq \sigma$ for any $u \in \Phi_{\lambda}^{m} \cap\left(S^{\delta} \backslash S^{\frac{\delta}{2}}\right)$ and $\lambda \geq \Lambda_{1}$.

Proof. We prove it by contradiction. Suppose that there exist a number $\delta_{0} \in\left(0, \tau_{1}\right)$, a positive sequence $\left\{\lambda_{j}\right\}$ with $\lambda_{j} \rightarrow 0$, and a sequence of function $\left\{u_{j}\right\} \subset \Phi_{\lambda_{j}}^{m} \cap\left(S^{\delta_{0}} \backslash S^{\frac{\delta_{0}}{2}}\right)$ such that

$$
\lim _{j \rightarrow+\infty} \Phi_{\lambda_{j}}^{\prime}\left(u_{j}\right)=0
$$

Up to a subsequence, we get $\left\{u_{j}\right\} \subset S^{\delta_{0}}$ and $\lim _{j \rightarrow \infty} \Phi_{\lambda_{j}}\left(u_{j}\right) \leq m$. By Lemma 5.3, we can deduce that there exists $u \in S$ such that $u_{j} \rightarrow u$ in $H_{\lambda_{j}}\left(\mathbb{R}^{2}\right)$. Therefore, $\operatorname{dist}_{\lambda_{j}}\left(u_{j}, S\right) \rightarrow 0$ as $j \rightarrow+\infty$. This contradict the assumption that $u_{j} \notin S^{\frac{\delta_{0}}{2}}$.

Lemma 5.5. There exist $\Lambda_{2} \geq \Lambda_{1}$ and $\alpha>0$ such that for any $\lambda \geq \Lambda_{2}$,

$$
\Phi_{\lambda}\left(y_{0}\left(t_{1}, t_{2}, t_{3}, t_{4}\right)\right) \geq m_{\lambda}-\alpha
$$

implies that $y_{0}\left(t_{1}, t_{2}, t_{3}, t_{4}\right) \in S^{\frac{\delta}{2}}$ for some $\delta \in\left(0, \tau_{1}\right)$.

Proof. We argue by contradiction. There exist $\lambda_{n} \rightarrow \infty, \alpha_{n} \rightarrow 0$ and $\left(t_{1}^{(n)}, t_{2}^{(n)}, t_{3}^{(n)}, t_{4}^{(n)}\right) \in\left[\frac{1}{2}, \frac{3}{2}\right]^{4}$ such that

$$
\Phi_{\lambda}\left(y_{0}\left(t_{1}^{(n)}, t_{2}^{(n)}, t_{3}^{(n)}, t_{4}^{(n)}\right)\right) \geq m_{\lambda_{n}}-\alpha_{n} \text { and } y_{0}\left(t_{1}^{(n)}, t_{2}^{(n)}, t_{3}^{(n)}, t_{4}^{(n)}\right) \notin S^{\frac{\delta}{2}} .
$$

We can choose a subsequence $\left(t_{1}^{(n)}, t_{2}^{(n)}, t_{3}^{(n)}, t_{4}^{(n)}\right) \rightarrow\left(\bar{t}_{1}, \bar{t}_{2}, \bar{t}_{3}, \bar{t}_{4}\right) \in\left[\frac{1}{2}, \frac{3}{2}\right]^{4}$. Then we have

$$
J\left(y_{0}\left(\bar{t}_{1}, \bar{t}_{2}, \bar{t}_{3}, \bar{t}_{4}\right)\right) \geq \lim _{n \rightarrow \infty}\left(m_{\lambda_{n}}-\alpha_{n}\right)=m \text {. }
$$

By the unique of 4-tuple, it is easy to have $\left(\bar{t}_{1}, \bar{t}_{2}, \bar{t}_{3}, \bar{t}_{4}\right)=(1,1,1,1)$. It implies that

$$
\lim _{n \rightarrow \infty}\left\|y_{0}\left(t_{1}^{(n)}, t_{2}^{(n)}, t_{3}^{(n)}, t_{4}^{(n)}\right)-y_{0}(1,1,1,1)\right\|=0 .
$$

Since $y_{0}(1,1,1,1)=v \in S$, which contradicts the assumption.

Now, we define

$$
\alpha_{0}:=\min \left\{\frac{a}{2}, \frac{\epsilon_{0}}{2}, \frac{1}{8} \delta \sigma^{2}\right\},
$$

where $\delta, \sigma, \alpha, \epsilon_{0}$ are from Lemma 5.4, Lemma 5.5 and Lemma 4.3-(iii) respectively. Using Lemma 4.2, one has that there exists $\Lambda_{3} \geq \Lambda_{2}$ such that

$$
\left|m_{\lambda}-m\right|<\alpha_{0} \text { for all } \lambda \geq \Lambda_{3} .
$$

Lemma 5.6. There exists a critical point $u_{\lambda}$ of $\Phi_{\lambda}$ with $u_{\lambda} \in S^{\delta} \cap \Phi_{\lambda}^{m}$ for $\lambda \geq \Lambda_{3}$.

Proof. We argue by contradiction. Fix a $\lambda \geq \Lambda_{3}$, by the Lemma 5.3, we can assume that there exists $0<\rho_{\lambda}<1$ such that $\left\|\Phi_{\lambda}^{\prime}(u)\right\| \geq \rho_{\lambda}$ on $S^{\delta} \cap \Phi_{\lambda}^{m}$. There exists a pseudo-gradient vector field $K_{\lambda}$ in $H_{\lambda}$ which is defined on a neighborhood $Z_{\lambda}$ of $S^{\delta} \cap \Phi_{\lambda}^{m}$ such that for any $u \in Z_{\lambda}$ there holds

$$
\left\{\begin{array}{l}
\left\|K_{\lambda}(u)\right\| \leq 2 \min \left\{1,\left\|\Phi_{\lambda}^{\prime}(u)\right\|\right\}, \\
\left\langle\Phi_{\lambda}^{\prime}(u), K_{\lambda}(u)\right\rangle \geq \min \left\{1,\left\|\Phi_{\lambda}^{\prime}(u)\right\|\right\}\left\|\Phi_{\lambda}^{\prime}(u)\right\| .
\end{array}\right.
$$


Define $\psi_{\lambda}$ be a Lipschitz function on $H_{\lambda}$ such that $0 \leq \psi_{\lambda} \leq 1, \psi_{\lambda} \equiv 1$ on $S^{\delta} \cap \Phi_{\lambda}^{m}$ and $\psi_{\lambda} \equiv 0$ on $H_{\lambda} \backslash Z_{\lambda}$. Define $\xi_{\lambda}$ be a Lipschitz function on $\mathbb{R}$ such that $0 \leq \xi_{\lambda} \leq 1, \xi_{\lambda}(t) \equiv 1$ if $\left|t-m_{\lambda}\right| \leq \frac{\alpha}{2}$ and $\xi_{\lambda}(t) \equiv 0$ if $\left|t-m_{\lambda}\right| \geq \alpha$. Let

$$
e_{\lambda}(u):=\left\{\begin{array}{l}
-\psi_{\lambda}(u) \xi_{\lambda}\left(\Phi_{\lambda}(u)\right) K_{\lambda}(u), \text { if } u \in Z_{\lambda}, \\
0, \text { if } u \in H_{\lambda} \backslash Z_{\lambda} .
\end{array}\right.
$$

Then there exists a global solution $\eta_{\lambda}: H_{\lambda} \times[0,+\infty) \rightarrow H_{\lambda}$ for the initial value problem

$$
\left\{\begin{array}{l}
\frac{d}{d \theta} \eta_{\lambda}(u, \theta)=e_{\lambda}\left(\eta_{\lambda}(u, \theta)\right) \\
\eta_{\lambda}(u, 0)=u
\end{array}\right.
$$

We can deduce that $\eta_{\lambda}$ has the following properties:

(i) $\eta_{\lambda}(u, \theta)=u$ if $\theta=0$ or $u \in H_{\lambda} \backslash Z_{\lambda}$ or $\left|\Phi_{\lambda}(u)-m_{\lambda}\right| \geq \alpha$;

(ii) $\left\|\frac{d}{d \theta} \eta_{\lambda}(u, \theta)\right\| \leq 2$;

(iii) $\frac{d}{d \theta} \Phi_{\lambda}\left(\eta_{\lambda}(u, \theta)\right)=\left\langle\Phi_{\lambda}^{\prime}\left(\eta_{\lambda}(u, \theta)\right), e_{\lambda}\left(\theta_{\lambda}(u, \theta)\right)\right\rangle \leq 0$.

Assertion 1. For any $\left(t_{1}, t_{2}, t_{3}, t_{4}\right) \in\left[\frac{1}{2}, \frac{3}{2}\right]^{4}$, there exists $\bar{\theta}=\theta\left(t_{1}, t_{2}, t_{3}, t_{4}\right) \in[0,+\infty)$ such that $\eta_{\lambda}\left(y_{0}\left(t_{1}, t_{2}, t_{3}, t_{4}\right), \bar{\theta}\right) \in \Phi_{\lambda}^{m_{\lambda}-\alpha_{0}}$.

Assuming by contradiction that there exists $\left(t_{1}, t_{2}, t_{3}, t_{4}\right) \in\left[\frac{1}{2}, \frac{3}{2}\right]^{4}$ such that

$$
\Phi_{\lambda}\left(\eta_{\lambda}\left(y_{0}\left(t_{1}, t_{2}, t_{3}, t_{4}\right), \theta\right)\right)>m_{\lambda}-\alpha_{0}
$$

for any $\theta \geq 0$. By Lemma 5.5, we get $y_{0}\left(t_{1}, t_{2}, t_{3}, t_{4}\right) \in S^{\frac{\delta}{2}}$. Note $\Phi_{\lambda}\left(y_{0}\left(t_{1}, t_{2}, t_{3}, t_{4}\right)\right) \leq m_{\lambda}+\alpha_{0}$, due the property (3) of $\eta_{\lambda}$,

$$
m_{\lambda}-\alpha_{0}<\Phi_{\lambda}\left(\eta_{\lambda}\left(y_{0}\left(t_{1}, t_{2}, t_{3}, t_{4}\right), \theta\right)\right)<\Phi_{\lambda}\left(y_{0}\left(t_{1}, t_{2}, t_{3}, t_{4}\right)\right) \leq m \leq m_{\lambda}+\alpha_{0} .
$$

So we can deduce that $\xi_{\lambda}\left(\Phi_{\lambda}\left(\eta_{\lambda}\left(y_{0}\left(t_{1}, t_{2}, t_{3}, t_{4}\right), \theta\right)\right)\right) \equiv 1$. If $\eta_{\lambda}\left(y_{0}\left(t_{1}, t_{2}, t_{3}, t_{4}\right), \theta\right) \in S^{\delta}$ for all $\theta \geq 0$, so it imply that

$$
\psi\left(\eta_{\lambda}\left(y_{0}\left(t_{1}, t_{2}, t_{3} . t_{4}\right), \theta\right)\right) \equiv 1 \text { and }\left\|\Phi_{\lambda}^{\prime}\left(\eta_{\lambda}\left(y_{0}\left(t_{1}, t_{2}, t_{3}, t_{4}\right), \theta\right)\right)\right\| \geq \rho_{\lambda} \text { for all } \theta>0 .
$$

It follows that

$$
\Phi_{\lambda}\left(\eta_{\lambda}\left(y_{0}\left(t_{1}, t_{2}, t_{3}, t_{4}\right), \frac{\alpha}{\rho_{\lambda}^{2}}\right)\right) \leq m_{\lambda}+\frac{\alpha}{2}-\int_{0}^{\frac{\alpha}{\rho_{\lambda}^{2}}} \rho_{\lambda}^{2} d t \leq m_{\lambda}-\frac{\alpha}{2} \leq m_{\lambda}-\alpha_{0},
$$

which is a contradiction. Thus, there exists $\theta_{3}>0$ such that $\eta_{\lambda}\left(y_{0}\left(t_{1}, t_{2}, t_{3}, t_{4}\right), \theta_{3}\right) \notin S^{\delta}$. Note that $y_{0}\left(t_{1}, t_{2}, t_{3}, t_{4}\right) \in S^{\frac{\delta}{2}}$, there exist $0<\theta_{1}<\theta_{2}<\theta_{3}$ such that

$$
\eta_{\lambda}\left(y_{0}\left(t_{1}, t_{2}, t_{3}, t_{4}\right), \theta_{1}\right) \in \partial S^{\frac{\delta}{2}}, \eta_{\lambda}\left(y_{0}\left(t_{1}, t_{2}, t_{3}, t_{4}\right), \theta_{2}\right) \in \partial S^{\delta}
$$

and $\eta_{\lambda}\left(y_{0}\left(t_{1}, t_{2}, t_{3}, t_{4}\right), \theta\right) \in S^{\delta} \backslash S^{\frac{\delta}{2}}$ for all $\theta \in\left(\theta_{1}, \theta_{2}\right)$. By Lemma 5.4, one has

$$
\left\|\Phi_{\lambda}^{\prime}\left(\eta_{\lambda}\left(y_{0}\left(t_{1}, t_{2}, t_{3}, t_{4}\right), \theta\right)\right)\right\| \geq \sigma \text { for all } \theta \in\left(\theta_{1}, \theta_{2}\right) .
$$

Using the property (2) of $\eta_{\lambda}$ we get that

$$
\frac{\delta}{2} \leq\left\|\eta_{\lambda}\left(y_{0}\left(t_{1}, t_{2}, t_{3}, t_{4}\right), \theta_{2}\right)-\eta_{\lambda}\left(y_{0}\left(t_{1}, t_{2}, t_{3}, t_{4}\right), \theta_{1}\right)\right\| \leq 2\left|\theta_{2}-\theta_{1}\right| .
$$

This deduce that

$$
\begin{aligned}
\Phi_{\lambda}\left(\eta_{\lambda}\left(y_{0}\left(t_{1}, t_{2}, t_{3}, t_{4}\right), \theta_{2}\right)\right) & \leq \Phi_{\lambda}\left(\eta_{\lambda}\left(y_{0}\left(t_{1}, t_{2}, t_{3}, t_{4}\right), 0\right)\right)+\int_{0}^{\theta_{2}} \frac{d}{d \theta} \Phi_{\lambda}\left(\eta_{\lambda}(u, v, \theta)\right) d \theta \\
& <\Phi_{y}\left(y_{0}\left(t_{1}, t_{2}, t_{3}, t_{4}\right)\right)+\int_{\theta_{1}}^{\theta_{2}} \frac{d}{d \theta} \Phi_{\lambda}\left(\eta_{\lambda}(u, v, \theta)\right) d \theta \\
& \leq m_{\lambda}+\alpha_{0}-\sigma^{2}\left(\theta_{2}-\theta_{1}\right) \leq m_{\lambda}+\alpha_{0}-\frac{1}{4} \delta \sigma^{2} \\
& \leq m_{\lambda}-\alpha_{0}
\end{aligned}
$$


which is a contradiction. Therefore, we prove the assertion 1.

Now, we define

$$
\Gamma\left(t_{1}, t_{2}, t_{3}, t_{4}\right):=\inf \left\{\theta \geq 0: \Phi_{\lambda}\left(\eta_{\lambda}\left(y_{0}\left(t_{1}, t_{2}, t_{3}, t_{4}\right), \theta\right)\right) \leq m_{\lambda}-\alpha_{0}\right\}
$$

and

$$
\tilde{y}\left(t_{1}, t_{2}, t_{3}, t_{4}\right):=\eta_{\lambda}\left(y_{0}\left(t_{1}, t_{2}, t_{3}, t_{4}\right), \Gamma\left(t_{1}, t_{2}, t_{3}, t_{4}\right)\right) .
$$

Then $\Phi_{\lambda}\left(\tilde{y}\left(t_{1}, t_{2}, t_{3}, t_{4}\right)\right) \leq m_{\lambda}-\alpha_{0}$ for all $\left(t_{1}, t_{2}, t_{3}, t_{4}\right) \in\left[\frac{1}{2}, \frac{3}{2}\right]^{4}$.

Assertion 2. $\tilde{y}\left(t_{1}, t_{2}, t_{3}, t_{4}\right)=\eta_{\lambda}\left(y_{0}\left(t_{1}, t_{2}, t_{3}, t_{4}\right), \Gamma\left(t_{1}, t_{2}, t_{3}, t_{4}\right)\right) \in \Sigma_{\lambda}$.

For any $\left(t_{1}, t_{2}, t_{3}, t_{4}\right) \in \partial\left[\frac{1}{2}, \frac{3}{2}\right]^{4}$, we have

$$
\Phi_{\lambda}\left(y_{0}\left(t_{1}, t_{2}, t_{3}, t_{4}\right)\right) \leq J\left(y_{0}\left(t_{1}, t_{2}, t_{3}, t_{4}\right)\right)<m-\epsilon_{0} \leq m_{\lambda}+\alpha_{0}-\epsilon_{0} \leq m_{\lambda}-\alpha_{0},
$$

which implies that $\Gamma\left(t_{1}, t_{2}, t_{3}, t_{4}\right)=0$. So $\tilde{y}\left(t_{1}, t_{2}, t_{3}, t_{4}\right)=y_{0}\left(t_{1}, t_{2}, t_{3}, t_{4}\right)$ for $\left(t_{1}, t_{2}, t_{3}, t_{4}\right) \in \partial\left[\frac{1}{2}, \frac{3}{2}\right]^{4}$. We also need to prove $\left\|\tilde{y}\left(t_{1}, t_{2}, t_{3}, t_{4}\right)\right\| \leq 6 \tau_{2}+\tau_{1}$ for all $\left[\frac{1}{2}, \frac{3}{2}\right]^{4}$ and $\Gamma\left(t_{1}, t_{2}, t_{3}, t_{4}\right)$ is continuous with respect to $\left(t_{1}, t_{2}, t_{3}, t_{4}\right)$.

For any $\left(t_{1}, t_{2}, t_{3}, t_{4}\right) \in\left[\frac{1}{2}, \frac{3}{2}\right]^{4}$, we have $\Gamma\left(t_{1}, t_{2}, t_{3}, t_{4}\right)=0$ if $\Phi_{\lambda}\left(y_{0}\left(t_{1}, t_{2}, t_{3}, t_{4}\right)\right) \leq m_{\lambda}-\alpha_{0}$, so $\tilde{y}\left(t_{1}, t_{2}, t_{3}, t_{4}\right)=y_{0}\left(t_{1}, t_{2}, t_{3}, t_{4}\right)$. By the definition of $y_{0}(t)$, we have $\left\|\tilde{y}\left(t_{1}, t_{2}, t_{3}, t_{4}\right)\right\| \leq 6 \tau_{2}<6 \tau_{2}+\tau_{1}$.

On the other hand, if $\Phi_{\lambda}\left(y_{0}\left(t_{1}, t_{2}, t_{3}, t_{4}\right)\right)>m_{\lambda}-\alpha_{0}$, it implies that $y_{0}\left(t_{1}, t_{2}, t_{3}, t_{4}\right) \in S^{\frac{\delta}{2}}$ and

$$
m_{\lambda}-\alpha_{0}<\Phi_{\lambda}\left(\eta_{\lambda}\left(y_{0}\left(t_{1}, t_{2}, t_{3}, t_{4}\right), \theta\right)\right)<m_{\lambda}+\alpha_{0} \text {, for all } \theta \in\left[0, \Gamma\left(t_{1}, t_{2}, t_{3}, t_{4}\right)\right) .
$$

So one has

$$
\xi_{\lambda}\left(\Phi_{\lambda}\left(\eta_{\lambda}\left(y_{0}\left(t_{1}, t_{2}, t_{3}, t_{4}\right), \theta\right)\right)\right) \equiv 1 \text { for all } \theta \in\left[0, \Gamma\left(t_{1}, t_{2}, t_{3}, t_{4}\right)\right) .
$$

Now, we will prove $\tilde{y}\left(t_{1}, t_{2}, t_{3}, t_{4}\right) \in S^{\delta}$. Otherwise, $\tilde{y}\left(t_{1}, t_{2}, t_{3}, t_{4}\right) \notin S^{\delta}$, similar to the proof of assertion 1 , we can find two constants $0<\theta_{1}<\theta_{2}<\Gamma\left(t_{1}, t_{2}, t_{3}, t_{4}\right)$ such that

$$
\Phi_{\lambda}\left(\eta_{\lambda}\left(y_{0}\left(t_{1}, t_{2}, t_{3}, t_{4}\right), \theta_{2}\right)\right)<m_{\lambda}-\alpha_{0} .
$$

It contradicts to the definition of $\Gamma\left(t_{1}, t_{2}, t_{3}, t_{4}\right)$. Therefore

$$
\tilde{y}\left(t_{1}, t_{2}, t_{3}, t_{4}\right)=\eta_{\lambda}\left(y_{0}\left(t_{1}, t_{2}, t_{3}, t_{4}\right), \Gamma\left(t_{1}, t_{2}, t_{3}, t_{4}\right)\right) \in S^{\delta} .
$$

Thus, there exists $u \in S$, such that

$$
\left\|\tilde{y}\left(t_{1}, t_{2}, t_{3}, t_{4}\right)\right\| \leq\|u\|+\tau_{1} \leq 6 \tau_{2}+\tau_{1} .
$$

To prove the continuity of $\Gamma\left(t_{1}, t_{2}, t_{3}, t_{4}\right)$, we fix arbitrarily $\left(t_{1}, t_{2}, t_{3}, t_{4}\right) \in\left[\frac{1}{2}, \frac{3}{2}\right]^{4}$. First, we assume that $\Phi_{\lambda}\left(\tilde{y}\left(t_{1}, t_{2}, t_{3}, t_{4}\right)\right)<m_{\lambda}-\alpha_{0}$. In this case, it is to see that $\Gamma\left(t_{1}, t_{2}, t_{3}, t_{4}\right)=0$, which gives that $\Phi_{\lambda}\left(y_{0}\left(t_{1}, t_{2}, t_{3}, t_{4}\right)\right)<m_{\lambda}-\alpha_{0}$. By the continuity of $y_{0}$, there exists $r>0$ such that for any $\left(s_{1}, s_{2}, s_{3}, s_{4}\right) \in$ $B_{r}\left(t_{1}, t_{2}, t_{3}, t_{4}\right) \cap\left[\frac{1}{2}, \frac{3}{2}\right]^{4}$, we have $\Phi_{\lambda}\left(y_{0}\left(s_{1}, s_{2}, s_{3}, s_{4}\right)\right)<m_{\lambda}-\alpha_{0}$, so $\Gamma\left(s_{1}, s_{2}, s_{3}, s_{4}\right)=0$, and hence $\Gamma$ is continuous at $\left(t_{1}, t_{2}, t_{3}, t_{4}\right)$. On the other hand, we assume that $\Phi_{\lambda}\left(\tilde{y}\left(t_{1}, t_{2}, t_{3}, t_{4}\right)\right)=m_{\lambda}-\alpha_{0}$. Similar to the proof of assertion 1 , we can deduce that $\tilde{y}\left(t_{1}, t_{2}, t_{3}, t_{4}\right)=\eta_{\lambda}\left(y_{0}\left(t_{1}, t_{2}, t_{3}, t_{4}\right), \Gamma\left(t_{1}, t_{2}, t_{3}, t_{4}\right)\right) \in S^{\delta}$, thus

$$
\left\|\Phi_{\lambda}^{\prime}\left(\eta_{\lambda}\left(y_{0}\left(t_{1}, t_{2}, t_{3}, t_{4}\right), \Gamma\left(t_{1}, t_{2}, t_{3}, t_{4}\right)\right)\right)\right\| \geq \rho_{\lambda}>0 \text {. }
$$

Therefore, for any $w>0$, we have

$$
\Phi_{\lambda}\left(\eta_{\lambda}\left(y_{0}\left(t_{1}, t_{2}, t_{3}, t_{4}\right), \Gamma\left(t_{1}, t_{2}, t_{3}, t_{4}\right)+w\right)\right)<m_{\lambda}-\alpha_{0} .
$$

Using the continuity of $\eta_{\lambda}$, there exists $r>0$ such that

$$
\Phi_{\lambda}\left(\eta_{\lambda}\left(y_{0}\left(s_{1}, s_{2}, s_{3}, s_{4}\right), \Gamma\left(t_{1}, t_{2}, t_{3}, t_{4}\right)+w\right)\right)<m_{\lambda}-\alpha_{0}
$$

for any $\left(s_{1}, s_{2}, s_{3}, s_{4}\right) \in B_{r}\left(t_{1}, t_{2}, t_{3}, t_{4}\right) \cap\left[\frac{1}{2}, \frac{3}{2}\right]^{4}$. Thus, $\Gamma\left(s_{1}, s_{2}, s_{3}, s_{4}\right) \leq \Gamma\left(t_{1}, t_{2}, t_{3}, t_{4}\right)+w$. It follows that

$$
0 \leq \limsup _{\left(s_{1}, s_{2}, s_{3}, s_{4}\right) \rightarrow\left(t_{1}, t_{2}, t_{3}, t_{4}\right)} \Gamma\left(s_{1}, s_{2}, s_{3}, s_{4}\right) \leq \Gamma\left(t_{1}, t_{2}, t_{3}, t_{4}\right) .
$$


If $\Gamma\left(t_{1}, t_{2}, t_{3}, t_{4}\right)=0$, we immediately obtain that

$$
\lim _{\left(s_{1}, s_{2}, s_{3}, s_{4}\right) \rightarrow\left(t_{1}, t_{2}, t_{3}, t_{4}\right)} \Gamma\left(s_{1}, s_{2}, s_{3}, s_{4}\right)=\Gamma\left(t_{1}, t_{2}, t_{3}, t_{4}\right) .
$$

If $\Gamma\left(t_{1}, t_{2}, t_{3}, t_{4}\right)>0$, we can similarly deduce that

$$
\Phi_{\lambda}\left(\eta_{\lambda}\left(y_{0}\left(s_{1}, s_{2}, s_{3}, s_{4}\right), \Gamma\left(t_{1}, t_{2}, t_{3}, t_{4}\right)-w\right)\right)>m_{\lambda}-\alpha_{0}
$$

for any $0<w<\Gamma\left(t_{1}, t_{2}, t_{3}, t_{4}\right)$.

By the continuity of $\eta_{\lambda}$ again, we see that

$$
\liminf _{\left(s_{1}, s_{2}, s_{3}, s_{4}\right) \rightarrow\left(t_{1}, t_{2}, t_{3}, t_{4}\right)} \Gamma\left(s_{1}, s_{2}, s_{3}, s_{4}\right) \geq \Gamma\left(t_{1}, t_{2}, t_{3}, t_{4}\right) .
$$

Combining (5.3) and (5.4), it is easy to see $\Gamma$ is continuous at $\left(t_{1}, t_{2}, t_{3}, t_{4}\right)$. This completes the proof of Assertion 2.

Thus, we have proved that $\tilde{y}\left(t_{1}, t_{2}, t_{3}, t_{4}\right) \in \Sigma_{\lambda}$ and

$$
\max _{\left(t_{1}, t_{2}, t_{3}, t_{4}\right) \in\left[\frac{1}{2}, \frac{3}{2}\right]^{4}} \Phi_{\lambda}\left(\tilde{y}\left(t_{1}, t_{2}, t_{3}, t_{4}\right)\right) \leq m_{\lambda}-\alpha_{0}
$$

which contradicts the definition of $m_{\lambda}$. This completes the proof.

Proof of Theorem 1.1 We still prove it with $T_{1}=\{1\}, T_{2}=\{2\}$ and $T_{3}=\{3\}$. By Lemma 5.6, when $\lambda>\Lambda_{3}$, we can get that there exists a solution $u_{\lambda} \in S^{\delta} \cap \Phi_{\lambda}^{m}$ for equation (3.3). By Lemma 3.2, we can know that $u_{\lambda}$ is a solution of equation (1.5) when $\lambda>\Lambda:=\max \left\{\Lambda_{0}, \Lambda_{3}\right\}$. Moreover, combining with Lemma 5.3, $u_{\lambda} \rightarrow u \in S$ (up to subsequence) strongly in $H_{r}^{1}\left(\mathbb{R}^{2}\right)$. So, we complete the proof of Theorem 1.1.

Acknowledgement: This work was supported by the NNSF (Nos. 11571370, 11601145, 11701173), by the Natural Science Foundation of Hunan Province (Nos. 2017JJ3130, 2017JJ3131), by the Excellent youth project of Education Department of Hunan Province (17B143, 17A113, 18B342), by the Hunan University of Commerce Innovation Driven Project for Young Teacher (16QD008), and by the Project of China Postdoctoral Science Foundation (2019M652790).

\section{References}

[1] C. O. Alves, Multiplicity of multi-bump type nodal solutions for a class of elliptic problems in $\mathbb{R}^{N}$, Topol. Methods Nonlinear Anal., 34 (2009) 231-250.

[2] C. O. Alves, D. S. Pereira, Multiplicity of multi-bump type nodal solutions for a class of elliptic problems with exponential critical growth in $\mathbb{R}^{2}$, Proc. Edin. Math. Soc., 60 (2017) 273-297.

[3] C. O. Alves, M. B. Yang, Existence of positive multi-bump solutions for a Schrödinger-Poisson system in $\mathbb{R}^{3}$, Discrete Contin. Dyn. Syst., 36 (2016) 5881-5910.

[4] C. O. Alves, A. B. Nóbrega, M. B. Yang, Multi-bump solutions for Choquard equation with deepening potential well, Calc. Var. (2016) 55:48, DOI 10.1007/s00526-016-0984-9.

[5] L. Bergé, A. de Bouard, J. C. Saut, Blowing up time-dependent solutions of the planar Chern-Simons gauged nonlinear Schrödinger equation, Nonlinearity 8 (1995) 235-253.

[6] J. Byeon, H. Huh, J. Seok, Standing waves of nonlinear Schrödinger equations with the gauge field, J. Funct. Anal. 263 (2012) 1575-1608.

[7] J. Byeon, H. Huh, J. Seok, On standing waves with a vortex point of order $N$ for the nonlinear Chern-Simons-Schrödinger equations, J. Diffe. Equ., 261 (2016) 1285-1316.

[8] H. Berestycki, P. L. Lions, Nonlinear scalar field equations. I. Existence of a ground state, Arch. Rational Mech. Anal., 82 (1983) 313-345.

[9] T. Bartsch, A. Pankov, Z. Q. Wang, Nonlinear Schrödinger equations with steep potential well, Commun. Contemp. Math. 3 (2001) 549-569.

[10] P. L. Cunha, P. D'avenia, A. Pomponio, G. Siciliano, A multiplicity result for Chern-Simons-Schrödinger equation with a general nonlinearity, Nonlinear Differ. Equ. Appl. 22 (2015) 1381-1850.

[11] X. W. Chen, P. Smith, On the unconditional uniqueness of solutions to the infinite radial Chern-Simons-chrödinger hierarchy, Anal. PDE 7 (2014) 1683-1712. 
[12] S. T. Chen, B. L. Zhang, X. H. Tang, Existence and concentration of semiclassical ground state solutions for the generalized Chern-Simons-Schrödinger system in $H^{1}\left(\mathbb{R}^{2}\right)$, Nonlinear Anal., 185 (2019) 68-96.

[13] S. T. Chen, X. H. Tang, Ground state solutions of Schrödinger-Poisson systems with variable potential and convolution nonlinearity, J. Math. Anal. Appl., 473 (2019) 87-111.

[14] V. Dunne, Self-Dual Chern-Simons Theories, Springer, 1995.

[15] Y. Ding, K. Tanaka, Multiplicity of positive solutions of a nonlinear Schrödinger equation, Manuscr. Math. 112 (2003) 109135.

[16] Y. B. Deng, S. J. Peng, W. Shuai, Nodal standing waves for a gauged nonlinear Schrödinger equation in $\mathbb{R}^{2}$, J. Diffe. Equ., 264 (2018) 4006-4035.

[17] M. del Pino, P. Felmer, Local Mountain Pass for semilinear elliptic problems in unbounded domains, Calc. Var. Partial Differ. Equ. 4 (1996) 121-137.

[18] Y. Deng, W. Shuai, Sign-changing multi-bump solutions for kirchhoff-type equation in $\mathbb{R}^{3}$, Discrete Contin. Dyn. Syst., 38 (2018) 3139-3168.

[19] H. Huh, Blow-up solutions of the Chern-Simons-Schrödinger equations, Nonlinearity 22 (2009) 967-974.

[20] H. Huh, Standing waves of the Schrödinger equation coupled with the Chern-Simons gauge field, J. Math. Phys. 53 (2012) 063702.

[21] H. Huh, Energy Solution to the Chern-Simons-Schrödinger equations, Abst. Appl. Anal. 2013, Article ID 590653.

[22] R. Jackiw, S. Y. Pi, Soliton solutions to the gauged nonlinear Schrödinger equations, Phys. Rev. Lett. 64 (1990) $2969-2972$.

[23] R. Jackiw, S. Y. Pi, Classical and quantal nonrelativistic Chern-Simons theory, Phys. Rev. D 42 (1990) 3500-3513.

[24] R. Jackiw, S. Y. Pi, Self-dual Chern-Simons solitons, Progr. Theoret. Phys. Suppl. 107 (1992) 1-40.

[25] Y. Jiang, A. Pomponio, D. Ruiz, Standing waves for a gauged nonlinear Schrödinger equation with a vortex point, Commun. Contemp. Math. 18 (2016) 1550074.

[26] B. Liu, P. Smith, Global wellposedness of the equivariant Chern-Simons-Schrödinger equation, preprint arXiv:1312.5567.

[27] B. Liu, P. Smith, D. Tataru, Local wellposedness of Chern-Simons-Schrödinger, Int. Math. Res. Notices. doi:10.1093/imrn/rnt161.

[28] Z. Liu, Z. Ouyang, J. Zhang, Existence and multiplicity of sign-changing standing waves for a gauged nonlinear Schrödinger equation in $\mathbb{R}^{2}$, arXiv:1811.05881v1.

[29] G. B. Li, X. Luo, Normalized solutions for the Chern-Simons-Schrödinger equation in $\mathbb{R}^{2}$, Ann. Acad. Sci. Fenn. Math. 42 (2017) 405-428.

[30] X. Luo, Multiple normalized solutions for a planar gauged nonlinear Schrödinger equation, Z. Angew. Math. Phys. (2018) 69: 58. https://doi.org/10.1007/s00033-018-0952-7.

[31] G. B. Li, X. Luo, W. Shuai, Sign-changing solutions to a gauged nonlinear Schrödinger equation, J. Math. Anal. Appl. 455 (2017) 1559-1578.

[32] A. Pomponio, D. Ruiz, A variational analysis of a gauged nonlinear Schrödinger equation, J. Eur. Math. Soc. 17 (2015) $1463-$ 1486.

[33] A. Pomponio, D. Ruiz, Boundary concentration of a gauged nonlinear Schrödinger equation on large balls, Calc. Var. Partial Differ. Equ. 53 (2015) 289-316.

[34] D. Ruiz, The Schrödinger-Poisson equation under the effect of a nonlinear local term, J. Funct. Anal. 237 (2006) 655-674.

[35] Y. Sato, K. Tanaka, Sign-changing multi-bump solutions for nonlinear Schrödinger equations with steep potential wells, Trans. Amer. Math. Soc., 361 (2009) 6205-6253.

[36] J. Sun, L. Li, M. Cenceli, B. Gabrovšek, Infinitely many sign-changing solutions for Kirchhoff type problems in $\mathbb{R}^{3}$, Nonlinear Anal., 186 (2019) 33-54.

[37] X. H. Tang, S. T. Chen, Ground state solutions of Nehari-Pohozăev type for Schrödinger-Poisson problems with general potentials, Discrete. Contin. Dyn. Syst., 37 (2017) 4973-5002.

[38] X. H. Tang, S. T. Chen, Ground state solutions of Nehari-Pohozăev type for Kirchhoff-type problems with general potentials, Calc. Var. PDE, 56 (2017) 1-25.

[39] X. H. Tang, J. Zhang, W. Zhang, Existence and concentration of solutions for the Chern-Simons-Schrödinger system with general nonlinearity, Results. Math. 71 (2017) 643-655.

[40] Y. Y. Wan, J. G. Tan, Standing waves for the Chern-Simons-Schrödinger systems without (AR) condition, J. Math. Anal. Appl. 415 (2014) 422-434.

[41] M. Willem, Minimax Theorems, Proress in Nonlinear Differential Equations and Their Applications 24, Birkhäuser, Boston, MA, 1996.

[42] W. Zhang, J. Zhang, H. Mi, On fractional Schrödinger equation with periodic and asymptotically periodic conditions, Comput. Math. Appl. 74 (2017) 1321-1332.

[43] J. Zhang, W. Zhang, X. Tang, Ground state solutions for Hamiltonian elliptic system with inverse square potential, Discrete Contin. Dyn. Syst., 37 (2017) 4565-4583.

[44] J. Zhang, X. Tang, F. Zhao, On multiplicity and concentration of solutions for a gauged nonlinear Schrödinger equation, Appl. Anal., DOI: 10.1080/00036811.2018.1553033.

[45] J. Zhang, W. Zhang, X. Xie, Infinitely many solutions for a gauged nonlinear Schrödinger equation, Appl. Math. Lett., 88 (2019) 21-27. 